\title{
Formation of localized structures in bistable systems through nonlocal spatial coupling. II. The nonlocal Ginzburg-Landau equation
}

\author{
Lendert Gelens,,${ }^{1,2}$ Manuel A. Matías, ${ }^{2}$ Damià Gomila, ${ }^{2}$ Tom Dorissen, ${ }^{1}$ and Pere Colet $^{2}$ \\ ${ }^{1}$ Applied Physics Research Group (APHY), Vrije Universiteit Brussel, Pleinlaan 2, 1050 Brussels, Belgium \\ ${ }^{2}$ IFISC, Instituto de Física Interdisciplinar y Sistemas Complejos (CSIC-UIB), Campus Universitat Illes Balears, \\ E-07122 Palma de Mallorca, Spain
}

(Received 2 May 2013; published 21 January 2014)

\begin{abstract}
We study the influence of a linear nonlocal spatial coupling on the interaction of fronts connecting two equivalent stable states in the prototypical 1-dimensional real Ginzburg-Landau equation. While for local coupling the fronts are always monotonic and therefore the dynamical behavior leads to coarsening and the annihilation of pairs of fronts, nonlocal terms can induce spatial oscillations in the front, allowing for the creation of localized structures, emerging from pinning between two fronts. We show this for three different nonlocal influence kernels. The first two, mod-exponential and Gaussian, are positive definite and decay exponentially or faster, while the third one, a Mexican-hat kernel, is not positive definite.
\end{abstract}

DOI: 10.1103/PhysRevE.89.012915

\section{INTRODUCTION}

We have shown recently [1] that a nonlocal interaction term can induce oscillatory tails in otherwise monotonic fronts connecting two equivalent homogeneous steady states (HSSs) in the Ginzburg-Landau equation (GLE) for a 1-dimensional (1D) real field. As a consequence, the interaction between a pair of fronts has an oscillatory dependence with the distance between the fronts with an exponentially decaying envelope. The oscillatory dependence allows for a pair of fronts to be pinned at specific distances determined by the tail profile. In particular, localized structures (LSs) can arise as a consequence of the pinning.

In [2], to which we refer as Part I here, we have presented a suitable framework to understand the effect of linear nonlocal spatial coupling on the shape of a class of fronts connecting two equivalent HSSs, making it possible to determine the parameter regions where fronts have an oscillatory profile. Here we apply this general framework to rationalize and extend the results advanced in [1] and elucidate the region in parameter space where LSs can exist for different forms of nonlocal interaction.

In particular, in Part I we considered 1D extended systems described by a real field with a nonlocal interaction term $s F(x, \sigma)$, in which $s$ is a parameter that controls the overall strength and sign of the coupling while $F(x, \sigma)$ is a linear function of the field. $F(x, \sigma)$ can be written as the convolution of a spatially nonlocal kernel $K_{\sigma}(x)$ with the field $A(x)$,

$$
F(x, \sigma)=\int_{-\infty}^{\infty} K_{\sigma}\left(x-x^{\prime}\right) A\left(x^{\prime}\right) d x^{\prime},
$$

where $\sigma$ is a parameter of the kernel. For nonlocal kernels that spatially decay to zero at large distances and we take $\sigma$ as the parameter that controls the spatial extension (width) of the coupling.

Nonlocality can also appear in nonlinear terms, as for instance in systems involving heat or particle transport [3], Bose-Einstein condensates [4], nonlinear optical systems [5,6], liquid crystals [7], ecological competition of individuals [8], or species [9]. In many instances, however, the linearization around a HSS will lead to an effective linear nonlocal term,
PACS number(s): 05.45.Yv, 05.65.+b, 89.75.-k, 42.65.Tg

so that one could proceed in a way similar to that described in this work.

Here we consider three different interaction kernels that illustrate the generality of the spatially nonlocal effects considered. Two of the kernels are positive definite, Gaussian and mod-exponential, while the third is a not positive definite, a Mexican-hat kernel. Table I gives the expression in real and Fourier space of the three kernels used in the present work, while Fig. 1 displays its shape. Moreover, these three kernels are relevant in different applications.

Spatially nonlocal interactions in which the spatial interaction kernel is positive definite are either attractive (activatory) or repulsive (inhibitory) depending on the sign of $s$. The most usual kernels of this type are the Gaussian and the exponential, which have been studied in several contexts like competition effects in ecology [8-10], nonlinear optics [5,11-13], reactiondiffusion systems [14], and neuroscience [15-17]. Physically, in reaction-diffusion systems a spatially nonlocal interaction with an exponential kernel arises whenever an adiabatic elimination of a fast diffusing substance is performed [18]. In optical systems with quadratic nonlinearities the interaction between the fundamental wave and its second harmonic can be understood as the propagation of the fundamental wave in a nonlocal nonlinear medium with a mod-exponential kernel whose width depends on diffraction and phase mismatch [5].

More general exponential decaying kernels with a variable exponent, including the exponential and Gaussian as particular cases, have been also considered [9]. In some instances it is possible to reconstruct an interaction kernel from experimental data, as done in Ref. [19] for a thermal nonlinear optical medium.

Kernels with both attractive and repulsive parts have been introduced in the context of neuroscience. Neurons are intrinsically discrete units, but one can make use of continuous neural field models that are coarse grained descriptions of the spatiotemporal evolution at the tissue level. These models, like the two-layer (one activatory and the other inhibitory) network Wilson-Cowan model [20] typically include nonlocal effects. A Mexican-hat kernel was introduced by Amari [21] to describe in terms of an effective field model a mixed 
TABLE I. Definition of the three different kernels $K_{\sigma}(x, \sigma)$ used in this work together with their Fourier transform $\hat{K}_{\sigma}(k, \sigma)$ : Gaussian, the mod-exponential, and Mexican-hat kernels.

\begin{tabular}{lccc}
\hline \hline & Gaussian & Mod-exponential & Mexican hat \\
\hline$K_{\sigma}(x) \frac{1}{\sqrt{2 \pi} \sigma} e^{-x^{2} / 2 \sigma^{2}}$ & $\frac{1}{4 \sigma} e^{-|x| / 2 \sigma}$ & $\frac{\sqrt{2}}{\sqrt{\pi} \sigma}\left(1-b \frac{x^{2}}{\sigma^{2}}\right) e^{-x^{2} / 2 \sigma^{2}}$ \\
$\hat{K}_{\sigma}(k)$ & $e^{-k^{2} \sigma^{2} / 2}$ & $\frac{1}{1+4 \sigma^{2} k^{2}}$ & $2\left[1+b\left(-1+\sigma^{2} k^{2}\right)\right] e^{-\sigma^{2} k^{2} / 2}$ \\
\hline \hline
\end{tabular}

population of activatory and inhibitory neurons. Since then, Mexican-hat kernels displaying local activation and lateral inhibition characteristics have been used extensively in neuroscience [15,17] and also in reaction diffusion systems [22]. The reverse situation, local inhibition and lateral excitation, has been also considered [15]. In some physical systems nonlocal response functions with attractive and repulsive parts arise as a result of two or more competing processes [6]. Examples include optical systems with third and quintic order nonlocal nonlinearities [23], Bose-Einstein condensates with contact and dipolar interactions [4], and nematic liquid crystals subject to a light beam with thermal and orientational nonlinear responses [7,24]. Here we study a kernel resulting from the combination of two Gaussians, as shown in Table I and Fig. 1(c), which also makes it possible to recover the results for a Gaussian kernel in the limit $b \rightarrow 0$.

The three kernels we consider as prototypical examples decay exponentially with distance. Nonlocal terms with other types of spatial dependence have also been considered in the literature, as for example periodic response functions which appear in optical systems with quadratic nonlinearities depending on the sign of the phase mismatch [5,25] or nonlocal functions that decay slower than exponentially [26]. We do not consider these cases explicitly in this work.

The paper is organized as follows. In Sec. II we briefly describe the GLE equation with nonlocal interaction. In Sec. III we discuss the moment expansion of the kernel. Sections IV, V,
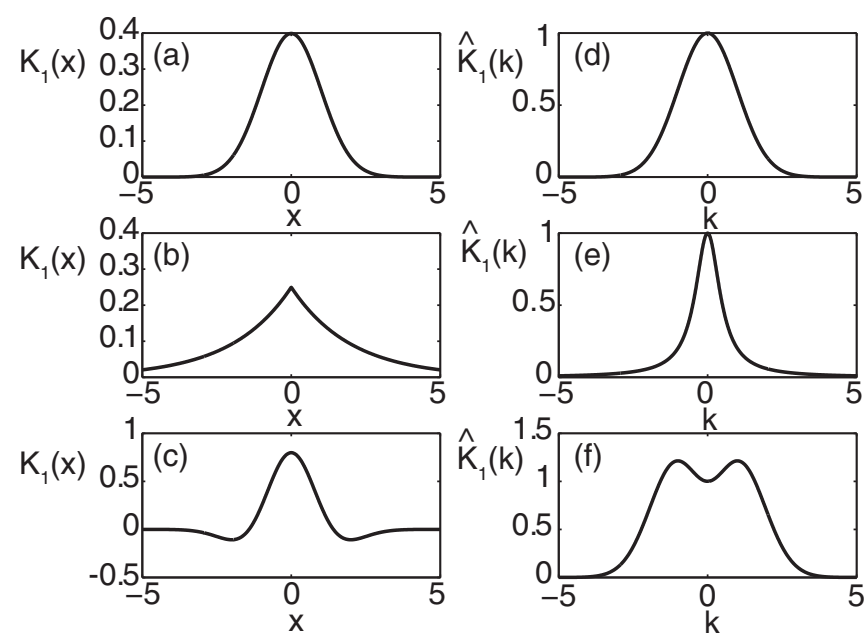

FIG. 1. Representations of the three nonlocal interaction kernels used in this work $(\sigma=1)$ : (a) Gaussian, (b) mod-exponential (Laplacian), (c) Mexican hat ( $b=1 / 2)$. The right panels (d)-(f) show the Fourier transforms of the kernels in panels (a)-(c), respectively. and VI are devoted to the Gaussian, mod-exponential, and Mexican-hat kernels, respectively. Finally, some concluding remarks are given in Sec. VII.

\section{THE GINZBURG-LANDAU EQUATION WITH NONLOCAL INTERACTION}

The prototypical cubic GLE for a real field $A$ in 1 spatial dimension can be written as [27]

$$
\partial_{t} A=\mu A-A^{3}+\partial_{x x} A .
$$

The parameter $\mu$ is the gain coefficient. The coefficients of the diffusion and cubic terms are set to one by suitably rescaling the spatial and temporal scales without loss of generality. The GLE is symmetric under the parity transformation $x \leftrightarrow-x$.

For $\mu<0$ the origin, $A_{s}=0$, is the only steady state (stable) in the system. At $\mu=0$ the system exhibits a pitchfork bifurcation, and two stable, symmetry related, HSSs appear at $A_{s}= \pm \sqrt{\mu}$. For $\mu>0$ the system is bistable and exhibits front solutions (kinks and antikinks) that connect the two HSSs. The fronts always decay to the HSS in a monotonic way.

We now consider an additional nonlocal term $F(x, \sigma)$ defined as in Eq. (1). We assume that the kernel is real and preserves the symmetry under the parity transformation $x \leftrightarrow-x$, namely $K_{\sigma}(x)=K_{\sigma}(-x)$. The extension of the GLE with nonlocal coupling can then be written as

$$
\partial_{t} A=\left(\mu-s M_{0}\right) A-A^{3}+\partial_{x x} A+s F(x, \sigma),
$$

where $s$ determines the strength of the nonlocal term. The term $-s M_{0} A$, where $M_{0}=\int_{-\infty}^{\infty} K_{\sigma}(x) d x$, compensates for the local contribution of $F(x, \sigma)$. Through this compensation the nonlocal system (3) has the same HSSs as the GLE with local coupling (2).

To analyze the linear stability of a HSS we consider perturbations from $A=A_{s}+\epsilon \exp (\Gamma t+i k x)$. Linearizing for small $\epsilon$, one obtains for Eq. (3) the dispersion relation

$$
\Gamma(k)=\mu^{\prime}-k^{2}+s\left[\hat{K}_{\sigma}(k)-M_{0}\right]
$$

where

$$
\mu^{\prime}=\mu-3 A_{s}^{2}
$$

and

$$
\hat{K}_{\sigma}(k)=\int_{-\infty}^{\infty} K_{\sigma}(x) e^{-i k x} d x
$$

is the Fourier transform of the kernel. Owing to the kernel symmetries, $\hat{K}_{\sigma}(k)=\hat{K}_{\sigma}(-k)$ is real valued and the dispersion relation $\Gamma(k)$ depends on $k$ only through $k^{2}=u$, and we can write

$$
\tilde{\Gamma}(u)=\mu^{\prime}-u+s\left[\tilde{\hat{K}}_{\sigma}(u)-M_{0}\right] .
$$

For $\mu \leqslant 0, A_{s}=0$ and $\mu^{\prime}=\mu$, while for $\mu>0, A_{s}= \pm \sqrt{\mu}$ and $\mu^{\prime}=-2 \mu<0$. As a consequence, all stable steady states of the GLE are associated with a negative value for $\mu^{\prime}$.

A given HSS becomes unstable if the maximum of $\Gamma(k)$ becomes positive at some $k_{c}$. If $k_{c}=0$ the instability is associated with a homogeneous perturbation, while if $k_{c} \neq 0$ the system undergoes a modulational instability (MI). For 
the local GLE, $s=0$, the dispersion relation has a parabolic shape with a single maximum at $k=0$ where $\Gamma(0)=\mu^{\prime}$. Changing the parameter $\mu$ the parabola moves rigidly in the vertical direction, so the maximum of the dispersion relation is always located at zero, and therefore none of the HSSs can undergo a MI. The zero HSS becomes unstable against homogeneous perturbations at $\mu=0$, where the two HSSs $A_{s}= \pm \sqrt{\mu}$ are born (pitchfork bifurcation). The two nonzero symmetric HSSs are always stable in the parameter region where they exist. As we see later, nonlocality induces a MI if, for some $k_{c}$, the last term in Eq. (4) overcomes the stabilizing $\mu^{\prime}-k^{2}$ term, making $\Gamma\left(k_{c}\right)=0$ for a finite $k_{c}$.

We note that the stability of a given HSS depends on the overall dispersion relation $\tilde{\Gamma}(u)$, which includes a constant term $\mu^{\prime}$. This term comes from linear gain or losses in the original GLE. In conserved systems, such as the nonlocal nonlinear Schrödinger equation, the overall dispersion relation does not have such a constant term [12]. Without $\mu^{\prime}$, all the terms in $\tilde{\Gamma}(u)$ are negative except for $s \tilde{\hat{K}}_{\sigma}(u)$. As a consequence, modulational instabilities can only appear for $s \tilde{\hat{K}}_{\sigma}(u)>0$; namely the sign of the nonlocal Kernel in Fourier space plays a critical role in the existence of instabilities as encountered in Ref. [12]. Here the constant term shifts the threshold for instabilities so that MIs can take place even for moderate negative values of $s \tilde{\hat{K}}_{\sigma}(u)$.

We focus now on stationary spatial structures. Setting the time derivative to zero in (3) one has

$$
\partial_{x x} A=\left(-\mu+s M_{0}\right) A+A^{3}-s F(x, \sigma) .
$$

Defining the intermediate variable $V$, one obtains the following 2D spatial dynamical system,

$$
A^{\prime}=V, \quad V^{\prime}=\left(-\mu+s M_{0}\right) A+A^{3}-s F(x, \sigma),
$$

where the prime stands for derivatives with respect to the spatial variable $x$. The fixed points of (9) correspond to solutions for $A$ which do not depend on $x$, thus to HSSs. Close to a HSS the shape of the fronts starting or ending at it can be obtained by considering a perturbation of the form $A(x)=A_{s}+\epsilon \exp (\lambda x)$ (where, in general, $\lambda$ is complex) and linearizing for small $\epsilon$. The spatial eigenvalues fulfill

$$
\Gamma_{s}(\lambda)=0
$$

where $\Gamma_{s}(\lambda)$ is the dispersion relation (4) replacing $k$ with a complex $-i \lambda$, namely,

$$
\Gamma_{s}(\lambda)=\Gamma(-i \lambda)=\mu^{\prime}+\lambda^{2}+s\left[\hat{K}_{\sigma}(-i \lambda)-M_{0}\right] .
$$

$\Gamma_{s}(\lambda)$ depends on $\lambda$ only through $\lambda^{2}=-u$; thus, spatial eigenvalues can also be obtained from $\tilde{\Gamma}\left(u_{0}\right)=0$. If $u_{0}$ is real, then there is a doublet of spatial eigenvalues $\lambda_{0}= \pm \sqrt{-u_{0}}$ with $\lambda_{0}$ real for $u_{0}<0$ or purely imaginary for $u_{0}>0$. If $u_{0}$ is complex, then $u_{0}^{*}$ is also a zero and therefore complex spatial eigenvalues come in quartets $\lambda_{0}= \pm q_{0} \pm i k_{0}$.

As discussed in Part I [2], if the eigenvalues are well separated, the leading eigenvalues, i.e., those with the smallest real part, determine the asymptotic approach to the HSS. If the leading eigenvalues are a real doublet, fronts approach monotonically to the HSS. If the leading eigenvalues are an imaginary doublet, the HSS is modulationally unstable. The most interesting case is when the leading eigenvalues are a complex quartet, since fronts starting or ending at the HSS have oscillatory tails and thus LSs may arise as a consequence of the tail interaction.

Varying parameters, two doublets can collide and lead to a complex quartet and vice versa. As explained in Part I the collision is signaled by a real double zero (RDZ) of $\tilde{\Gamma}(u)$, namely $\tilde{\Gamma}\left(u_{c}\right)=\tilde{\Gamma}^{\prime}\left(u_{c}\right)=0$ for $u_{c} \in R$. If $u_{c}>0$ two imaginary doublets become a complex quartet signaling a Hamiltonian-Hopf $(\mathrm{HH})$ bifurcation. If the $\mathrm{HH}$ occurs at the maximum of $\Gamma(k)$ it corresponds to a MI. If $u_{c}<0$ two real doublets become a complex quartet which corresponds to the so-called Belyakov-Devaney (BD) transition [28]. Moving on top of the RDZ manifold $u_{c}$ changes value and eventually can change sign, so that a $\mathrm{HH}$ becomes a BD and vice versa. This happens when $\tilde{\Gamma}(0)=\tilde{\Gamma}^{\prime}(0)=0$ and corresponds to a quadruple zero $(\mathrm{QZ})$ of the dispersion relation when written as function of $k, \Gamma(k)[2]$.

As shown in Part I, besides the QZ, there are two other codimension 2 (codim-2) points that play a relevant role in organizing the phase space dynamics, namely the cusp point where two BD or two HH manifolds start (or end) and the 3DZ $(i \omega)$ in which the HSS becomes simultaneously unstable to homogeneous and finite wavelength perturbations.

\section{MOMENT EXPANSION}

A qualitative understanding of the effects of a nonlocal coupling by means of a moment expansion is possible for kernels that in Fourier space have no singularities at finite distances, as is the case of the Gaussian kernel to be considered in Sec. IV. Proceeding as indicated in Part I, the nonlocal interaction can be written as a series of spatial derivatives of $A$ (see also [29]),

$$
F(x, \sigma)=\sum_{j=0}^{\infty} \frac{M_{2 j}}{(2 j) !} \frac{\partial^{2 j} A}{\partial x^{2 j}},
$$

where $M_{j}=\int_{-\infty}^{\infty} x^{j} K_{\sigma}(x) d x$. To describe the BD and $\mathrm{HH}$ transitions which involve four spatial eigenvalues, we need to keep the expansion terms at least up to fourth order derivatives.

For the GLE (3) truncating the expansion of the kernel at fourth order one has

$$
\partial_{t} A=\mu A-A^{3}+\left(1+\frac{1}{2 !} s M_{2}\right) \nabla^{2} A+\frac{1}{4 !} s M_{4} \nabla^{4} A
$$

Equation (13) is related to the widely studied Swift-Hohenberg equation [30,31]. An analogous truncation for a spatially nonlocal interaction was considered in Ref. [32]. Notice that Eq. (13) only makes sense if $s M_{4}<0$ since otherwise large wave number perturbations will always be amplified, leading to divergences. Table II gives the values of the moments for the nonsingular kernels considered in this article.

The dispersion relation for the fourth order truncated moment expansion is

$$
\tilde{\Gamma}(u)=\mu^{\prime}-\frac{2+s M_{2}}{2} u+\frac{s M_{4}}{24} u^{2} .
$$


TABLE II. First moments $M_{i}$ for the Gaussian and the Mexicanhat kernels.

\begin{tabular}{lcc}
\hline \hline Moment & Gaussian & Mexican hat \\
\hline$M_{0}$ & 1 & $2(1-b)$ \\
$M_{2}$ & $\sigma^{2}$ & $2(1-3 b) \sigma^{2}$ \\
$M_{4}$ & $3 \sigma^{4}$ & $6(1-5 b) \sigma^{4}$ \\
$M_{6}$ & $15 \sigma^{6}$ & $30(1-7 b) \sigma^{6}$ \\
$M_{8}$ & $105 \sigma^{8}$ & $210(1-9 b) \sigma^{8}$ \\
\hline \hline
\end{tabular}

The spatial eigenvalues are

$$
\begin{aligned}
u_{0} & =-\lambda_{0}^{2} \\
& =6 \frac{2+s M_{2} \pm \sqrt{\left(2+s M_{2}\right)^{2}-2 \mu^{\prime} s M_{4} / 3}}{s M_{4}} .
\end{aligned}
$$

The RDZ manifold of $\tilde{\Gamma}(u)$, which signals HH and BD transitions, is given by $\tilde{\Gamma}\left(u_{c}\right)=\tilde{\Gamma}^{\prime}\left(u_{c}\right)=0$ :

$$
\begin{gathered}
4 \mu^{\prime}-\left(2+s M_{2}\right) u_{c}=0, \\
s M_{4} u_{c}=6\left(2+s M_{2}\right) .
\end{gathered}
$$

Combining these two equations in order to eliminate $u_{c}$ yields the RDZ manifold,

$$
\mu_{\mathrm{RDZ}}^{\prime}=\frac{3\left(2+s M_{2}\right)^{2}}{2 s M_{4}},
$$

which is of codim-1 in the $3 \mathrm{D}\left(\mu^{\prime}, s M_{2}, s M_{4}\right)$ parameter space. Since we are considering $s M_{4}<0, \mu_{\mathrm{RDZ}}^{\prime}$ is always negative. Setting $u_{c}=0$ in (16) and (17), one obtains the QZ manifold

$$
\mu_{\mathrm{QZ}}^{\prime}=0, \quad s_{\mathrm{QZ}}=-2 / M_{2} \text {. }
$$

Since $\tilde{\Gamma}^{\prime \prime}(0)<0$, following the notation of Part I this is a $\mathrm{QZ}^{-}$point. For a fixed $s M_{4}$, considering the $\left(s, \mu^{\prime}\right)$ parameter space the RDZ manifold has the shape of a parabola with vertex at the QZ point and unfolding towards negative $\mu^{\prime}$. In the part of the RDZ with $s<s_{\mathrm{QZ}}, u_{c}>0$ and corresponds to a $\mathrm{HH}$ which in this case is a MI, while the other part corresponds to a BD. For parameter values in the region between the BD and MI lines, where the leading eigenvalues are a complex quartet, fronts connecting two equivalent homogeneous solutions have oscillatory tails and LSs can be formed.

In some cases, one is interested in the effect of the nonlocality for fixed values of the parameters of the local GLE, namely for a given $\mu^{\prime}$; then it is convenient to rewrite Eq. (18) so that $s$ is isolated,

$$
s_{\mathrm{RDZ}}=\frac{\mu^{\prime} M_{4}-6 M_{2} \pm \sqrt{\mu^{\prime 2} M_{4}^{2}-12 \mu^{\prime} M_{2} M_{4}}}{3 M_{2}^{2}},
$$

where the + solution corresponds to the MI transition and the - to the BD.

For kernels whose moments can be written as $M_{j}=\sigma^{j} \mathcal{M}_{j}$ (cf. Sec. III A of Part I), Eq. (20) becomes

$$
s_{\mathrm{RDZ}}=\frac{1}{3 \mathcal{M}_{2}}\left[\mu^{\prime} \frac{\mathcal{M}_{4}}{\mathcal{M}_{2}}-\frac{6}{\sigma^{2}} \pm \sqrt{\mu^{\prime 2} \frac{\mathcal{M}_{4}^{2}}{\mathcal{M}_{2}^{2}}-12 \mu^{\prime} \frac{\mathcal{M}_{4}}{\mathcal{M}_{2} \sigma^{2}}}\right] \text {. }
$$

In the limit of nonlocal interaction range going to zero, $\sigma \rightarrow 0$, one has $s_{\mathrm{RDZ}} \rightarrow-2 /\left(\mathcal{M}_{2} \sigma^{2}\right)$ for both BD and MI transitions. For infinite-range nonlocality, $\sigma \rightarrow \infty, s_{\mathrm{RDZ}} \rightarrow 0$ for the BD transition while $s_{\mathrm{RDZ}} \rightarrow 2 \mu^{\prime} \mathcal{M}_{4} /\left(3 \mathcal{M}_{2}^{2}\right)$ for the MI. These predictions will be compared with the results for a Gaussian kernel in the next section.

\section{THE GAUSSIAN KERNEL}

In this section we analyze the influence of a nonlocal Gaussian kernel in the shape of the front starting (or ending) at an HSS of the GLE. Without loss of generality this kernel can be normalized so that $M_{0}=1$. In terms of $u=-\lambda^{2}$ the dispersion relation obtained linearizing around the HSS can be written as (cf. Table I)

$$
\tilde{\Gamma}(u)=\mu^{\prime}-s-u+s \exp \left(-\sigma^{2} u / 2\right)
$$

The spatial eigenvalues are the zeros of (22), a transcendental equation that can be solved analytically in terms of the Lambert's $W$ function [see Eq. (A5) in the Appendix]. The result is

$$
\begin{aligned}
u_{0} & =-\lambda_{0}^{2} \\
& =\mu^{\prime}-s+\frac{2}{\sigma^{2}} W_{l}\left[\frac{s \sigma^{2}}{2} \exp \left(\frac{\sigma^{2}}{2}\left(-\mu^{\prime}+s\right)\right)\right],
\end{aligned}
$$

where $l \in \mathbb{Z}$ and $W_{l}(x)$ is the $l$ th branch of Lambert's $W$ function, $W(x) \in \mathbb{C}$, and, thus, the spectrum of spatial eigenvalues is infinite (numerable). The function $W$ has two real branches, $l=0$ and $l=-1$.

In order to determine the location of the MI and BD instabilities of the HSSs, we look for the RDZ of (22), which is given by $\tilde{\Gamma}\left(u_{c}\right)=\tilde{\Gamma}^{\prime}\left(u_{c}\right)=0$ :

$$
\begin{gathered}
\exp \left(-\sigma^{2} u_{c} / 2\right)=-\frac{2}{s \sigma^{2}}, \\
\mu^{\prime}=s+\frac{2}{\sigma^{2}}+u_{c} .
\end{gathered}
$$

One consequence of (24) is that BD and MI transitions require that $s<0$, as $u_{c}$ has to be real. Combining (24) and (25) to eliminate $u_{c}$ leads to the condition defining the RDZ manifold of $\tilde{\Gamma}(u)$. This manifold has one dimension less than the dimensionality of the parameter space. Since we have three parameters, $\mu^{\prime}, s$, and $\sigma$, the RDZ manifold is a 2D manifold given by

$$
\frac{s \sigma^{2}}{2} \exp \left[\frac{\sigma^{2}}{2}\left(-\mu^{\prime}+s\right)\right]=-\frac{1}{e} .
$$

The RDZ manifold given by (26) corresponds in Eq. (23) to the branching point of the two real branches of Lambert's $W$ function (see the Appendix), in which these two branches both merge and finish. Solving Eq. (26) for $\mu^{\prime}=\mu_{\mathrm{RDZ}}^{\prime}(s, \sigma)$, one has

$$
\mu_{\mathrm{RDZ}}^{\prime}=s+\frac{2}{\sigma^{2}}\left[1+\ln \left(-\frac{s \sigma^{2}}{2}\right)\right] .
$$

A cut of the $\mu_{\mathrm{RDZ}}^{\prime}$ manifold for $\sigma=2$ is shown in solid lines in Fig. 2.

The codim-2 QZ manifold (which is a line in our 3D parameter space), can be obtained by setting $u_{c}=0$ in (24) 


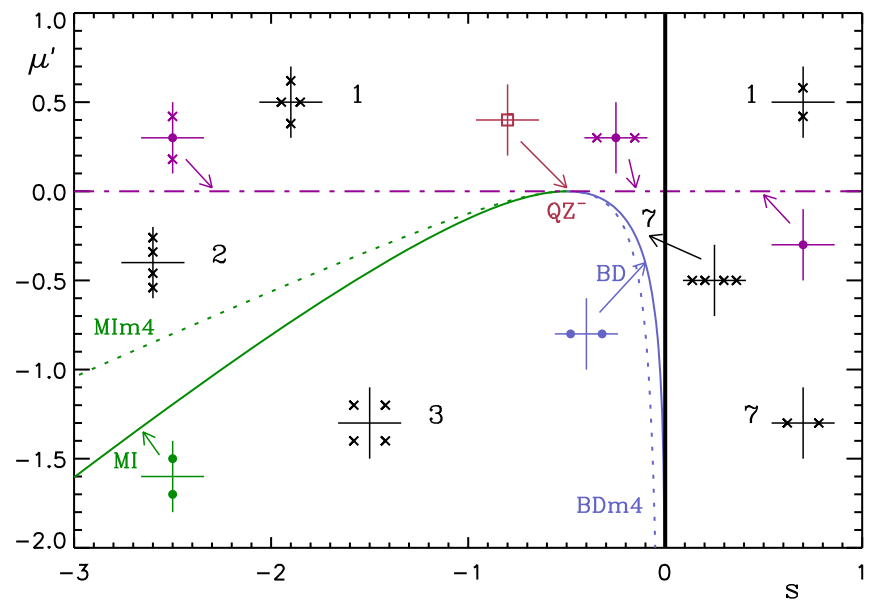

FIG. 2. (Color online) Boundaries in the $\left(s, \mu^{\prime}\right)$ plane at which the leading spatial eigenvalues of the GLE with a Gaussian kernel exhibit different transitions for $\sigma=2$. Sketches indicate the location of the leading eigenvalues in the $[\operatorname{Re}(\lambda), \operatorname{Im}(\lambda)]$ plane $(x$, signal simple eigenvalues; $\bullet$, double eigenvalues; and $\square$, quadruple eigenvalues). Dotted lines labeled as MIm4 and BDm4 show the MI and BD transitions given by the fourth moment expansion (18).

and (25) or, alternatively, locating the submanifold of RDZ (26) in which $u_{c}=0$. The result is that the QZ manifold is defined by

$$
\mu_{\mathrm{QZ}}^{\prime}=0, \quad s_{\mathrm{QZ}}=-2 / \sigma^{2} .
$$

For the parameters of the QZ, $\tilde{\Gamma}^{\prime \prime}(0)<0$; therefore, in the notation of Part I, this is a $\mathrm{QZ}^{-}$point. In the $\left(s, \mu^{\prime}\right)$ plane shown in Fig. 2 the QZ point is located at $(-1 / 2,0)$. The character of the two pieces of the RDZ manifold, BD or MI, can be elucidated by calculating $u_{c}$ on top of the manifold, such that, respectively, $u_{c}<0$ and $u_{c}>0$. From (24) one obtains

$$
u_{c}=\frac{2}{\sigma^{2}} \ln \left(-\frac{s \sigma^{2}}{2}\right) \text {. }
$$

Therefore, the part of the RDZ manifold in which $s \sigma^{2}<-2$ has a positive $u_{c}$ and corresponds to a MI while the part in which $-2<s \sigma^{2}<0$ corresponds to a BD. In Fig. 2 the MI is located at the left of the QZ point and the BD at the right. Fronts starting (or ending) at the HSS have oscillatory tails for parameter values in the region between the MI and the BD lines. This region is labeled as 3 in Fig. 2, in agreement with the notation used in Part I. The other parameter regions of the figure are also labeled as in Part I. For $s<0$ we refer to Part I for a detailed description of the regions and the transitions between them. The $s=0$ line corresponds to the GLE with local coupling for which there are only two spatial eigenvalues which are a real doublet for $\mu^{\prime}<0$ and an imaginary doublet for $\mu^{\prime}>0$. At $\mu=0$ the two components of the doublet collide at the origin (Hamiltonian-pitchfork bifurcation). For $s>0$, despite the presence of the Gaussian nonlocal kernel, the spatial dynamics shows a qualitative behavior similar to that for $s=0$.

The second derivative of $\tilde{\Gamma}(u)$, given by

$$
\tilde{\Gamma}^{\prime \prime}(u)=\frac{s \sigma^{4}}{4} \exp \left(-u \sigma^{2} / 2\right),
$$

does not vanish for any value of $u$ provided $s \neq 0$. This indicates that there is no cusp point for the GLE with a Gaussian nonlocal kernel. For $s=0$ the second derivative vanishes but this corresponds to the GLE with only local interaction whose dispersion relation is linear in $u$, thus it has nothing to do with a cusp point.

We now look for 3DZ and 3DZ $(i \omega)$ codim-2 points which, as discussed in Part I, correspond to the coincidence of a simple zero at the origin $\tilde{\Gamma}(0)=0$ and a RDZ at finite distance, $\tilde{\Gamma}\left(u_{c}\right)=\tilde{\Gamma}^{\prime}\left(u_{c}\right)=0$. The first condition, $\tilde{\Gamma}(0)=0$ implies $\mu^{\prime}=0$; thus, 3DZ and 3DZ $(i \omega)$ can be obtained setting $\mu_{\mathrm{RDZ}}^{\prime}=0$ in Eq. (27) and looking for solutions with nonzero $u_{c}$. There is no such a solution and therefore the GLE with a Gaussian nonlocal kernel does not have any $3 \mathrm{DZ}(i \omega)$ or $3 \mathrm{DZ}$ points.

The absence of cusp and 3DZ points indicates that the GLE with a Gaussian nonlocal kernel does not have any crossover manifold. This has strong implications on the location of the complex quartets in the $[\operatorname{Re}(\lambda), \operatorname{Im}(\lambda)]$ plane. In particular, if a real doublet is leading the spatial dynamics, changing parameters complex quartets cannot overcome the real doublet. In this case, the only way oscillatory tails can appear is after a BD transition in which two real doublets collide to become a complex quartet.

We now consider the effect of the nonlocal Gaussian kernel for given values of the parameters of the local dynamics, namely for a given $\mu^{\prime}$. Solving (26), e.g., for $s_{\mathrm{RDZ}}\left(\mu^{\prime}, \sigma\right)$, one obtains

$$
s_{\mathrm{RDZ}}=\frac{2}{\sigma^{2}} W_{l}\left[-\exp \left(\frac{\sigma^{2}}{2} \mu^{\prime}-1\right)\right] .
$$

Since the argument of $W$ is in the interval $[-1 / e, 0]$ the $s_{\mathrm{RDZ}}$ manifold has two branches corresponding to $l=0$ and $l=-1$. These two pieces of the RDZ manifold are organized by the codim-2 QZ manifold (28). Figure 3 shows three cuts of the $s_{\mathrm{RDZ}}\left(\mu^{\prime}, \sigma\right)$ for different values of $\mu^{\prime}$. At $\mu^{\prime}=0$ one has the QZ manifold (28) [Fig. 3(a)], from which the BD and MI branches emerge as $\mu^{\prime}$ is decreased [see panel (b)]. The upper branch has $u_{c}<0$ and therefore it corresponds to a BD while the lower branch corresponds to the MI. LSs exist for parameter values in the region between the BD and the MI curves. The BD and MI branches separate as $\mu^{\prime}$ is further decreased [see panel (c)]; thus, the region where LSs exists becomes larger. The asymptotic limit of both transitions as $\sigma \rightarrow \infty$ is $s_{\mathrm{BD}}(\sigma \rightarrow$ $\infty) \rightarrow 0^{-}$and $s_{\mathrm{MI}}(\sigma \rightarrow \infty) \rightarrow \mu^{\prime}[33]$.

To illustrate the main behaviors exhibited by the system, we plot in Fig. 4 the location in the complex $\lambda$ plane of the first few spatial eigenvalues of (23) for $\mu^{\prime}=-6$, three values of $s$ (at different rows) and three values $\sigma$ (at different columns). For attractive nonlocal interaction, $s>0$, only the principal branch, $W_{0}$, is real; thus, the spectrum contains only one real doublet [panels (a)-(c)]. For small $\sigma$ it is located very close to the real doublet of the local dynamics, as shown in panel (a). There is also an infinite number of complex eigenvalues but they are located outside the region shown in panel (a). As $\sigma$ increases, the location of the spatial eigenvalues approaches the imaginary axis. Since, as discussed before, the GLE with Gaussian kernel has no crossover manifolds, the real doublet is always the eigenvalue located closer to the imaginary axis [see panels (b) and (c)]. Neither a BD transition can exist for 

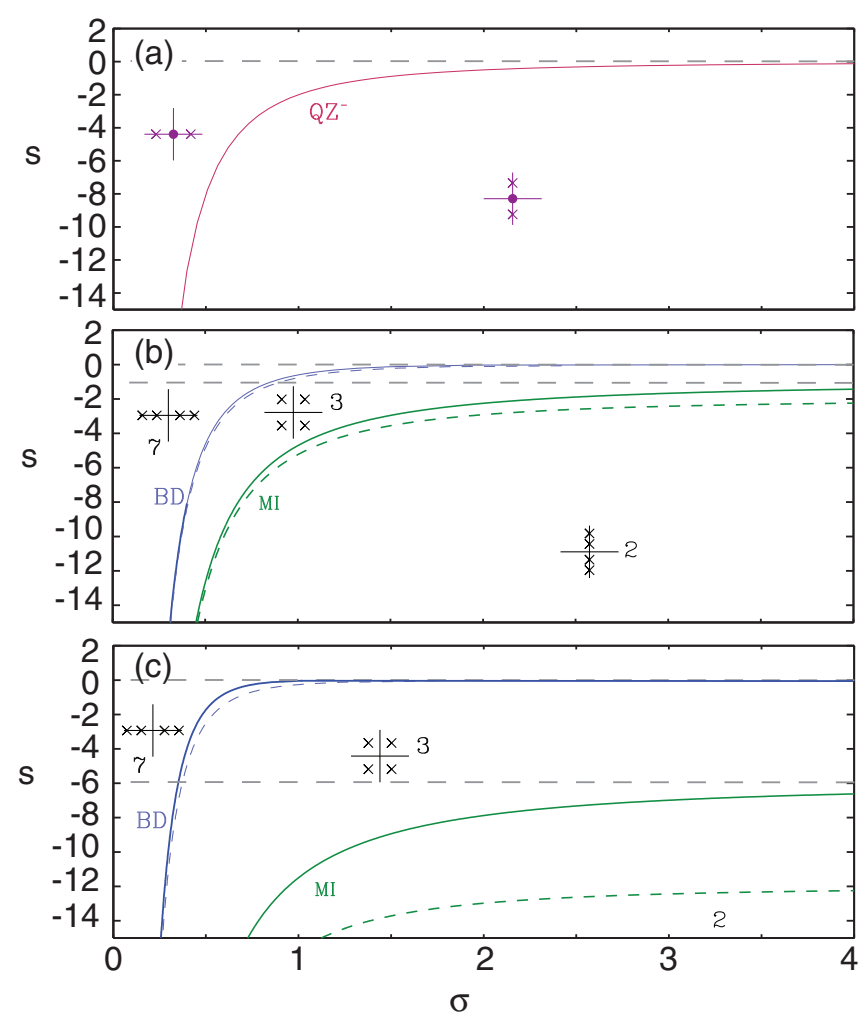

FIG. 3. (Color online) Boundaries in the $(\sigma, s)$ plane at which the leading spatial eigenvalues of the GLE with a Gaussian kernel exhibit different transitions. Panel (a) shows the QZ manifold at $\mu^{\prime}=0$. Panels (b) and (c) show the BD and MI manifolds at $\mu^{\prime}=-1$ and $\mu^{\prime}=$ -6 , respectively. The short-dashed curves show the BD and MI transitions as predicted by the fourth moment expansion [Eq. (21)]. Longdashed horizontal lines show the asymptotic values for $\sigma \rightarrow \infty$. Sketches represent the location of the leading eigenvalues.

$s>0$ because there is no other real doublet with which the leading real doublet can collide. As a consequence for $s>0$ the spatial dynamics is always led by a real doublet and fronts decay monotonically.

For repulsive nonlocal interaction, $s<0$, and small $\sigma$, the argument of $W_{l}(x)$ in (23) is in the range $x \in[-1 / e, 0]$, and both $W_{0}(x)$ and $W_{-1}(x)$ are real (see the Appendix) and, as a consequence, there are two pairs of real eigenvalues. The pair located closer to the origin is shown in panel (d) for $s=-1$ and in panel (g) for $s=-7.5$. Increasing $\sigma$, the two real doublets approach each other and collide at the BD transition, which corresponds to the branching point of $W$, beyond which there is no real solution. Panels (e) and (h) correspond to parameters at the right of the BD curve in Fig. 3, and one finds a leading complex quartet. Figure 5 illustrates the change of the front profile when crossing the BD line. From Eq. (26) one obtains that for $\mu^{\prime}=-6$ and $s=-1$ the BD line is located at $\sigma=\sqrt{(2 / 5) W_{0}(5 / e)} \approx 0.5708076$. For $\sigma=0.5$, at the left of the BD line, the fronts are monotonic. Figure 5(a) shows the detailed shape of the front close to the HSS corresponding to $A_{s}=\sqrt{\mu}$. The overall profile of the front connecting the two HSS is shown in the inset. Close to the HSS the front is well described by an exponential of the form

$$
A(x)-A_{s} \approx c_{1} e^{q_{1} x},
$$
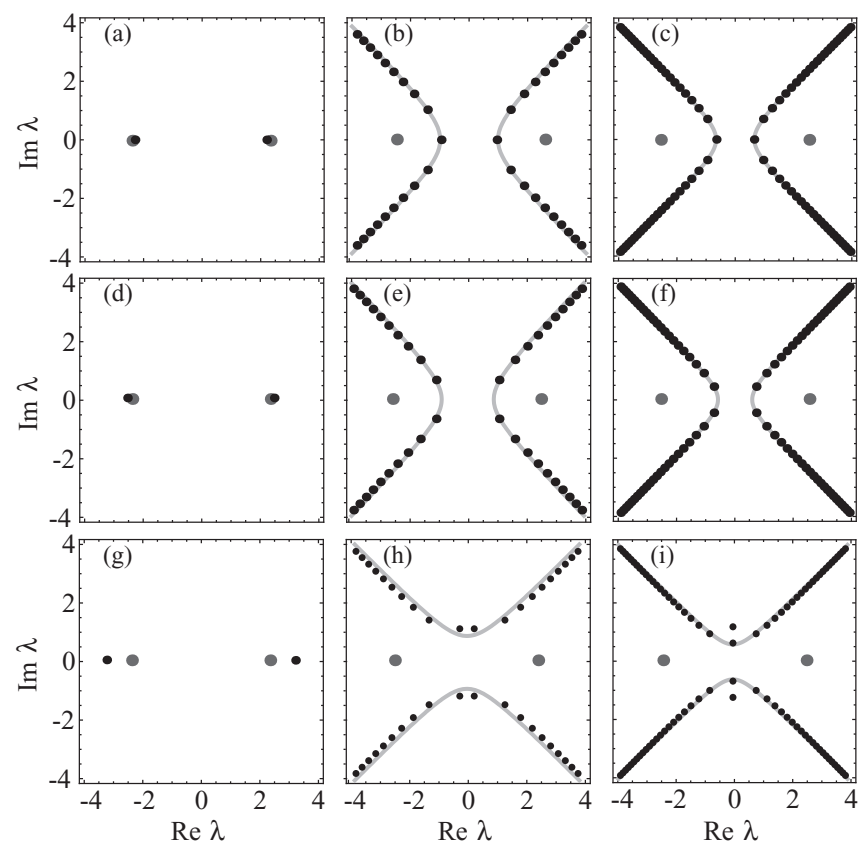

FIG. 4. Location of the first spatial eigenvalues for the GLE with a Gaussian nonlocal kernel in the complex $\lambda$ plane (shown as black dots) for $\mu^{\prime}=-6$. For comparison, the two gray dots show the location of the eigenvalues for the local GLE $(s=0)$. The hyperbola, given by Eq. (36), in grayscale represents an approximation for the location of the spatial eigenvalues. For panels (a)-(c) on the top row, $s=1$, for (d)-(f) on the middle row $s=-1$ and for (g)-(i) on the bottom row $s=-7.5$. For panels (a), (d), and (g) on the left column $\sigma=0.3$; for (b), (e), and (h) on the middle column $\sigma=2$; and for (c), (f), and (i) on the right column $\sigma=3$. For all values of $\sigma$ the number of spatial eigenvalues is infinite: The plot just presents the region around the origin in the complex plane.

where $q_{1}=-2.753$ is the leading spatial eigenvalue and the coefficient $c_{1}$ has been fitted to $c_{1}=-4.507$. When crossing the BD line oscillations in the front profile appear initially with an infinite wavelength. The front profile for $\sigma=1$ is shown in Fig. 5(b). Again close to the HSS the front profile is very well
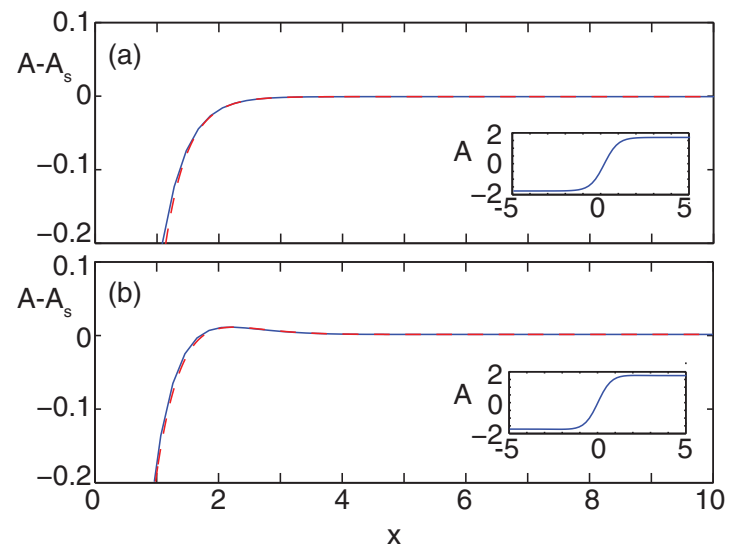

FIG. 5. (Color online) The solid line shows front profile for the GLE with a Gaussian nonlocal kernel for $\mu^{\prime}=-6, s=-1$, and (a) $\sigma=0.5$ and (b) $\sigma=1$. The red dashed lines show the approximations given by Eqs. (32) and (33) (see text). 
described by an exponential of the form

$$
A(x)-A_{s} \approx c_{1} e^{q_{1} x} \cos \left(k_{1} x+\phi_{1}\right),
$$

where $q_{1}=-2.01$ and $k_{1}=1.01$ are the real and imaginary part of the spatial eigenvalue and the coefficients $c_{1}=2.02$ and $\phi_{1}=2.91$ have been fitted.

Returning to the description of Fig. 4, once crossed the $\mathrm{BD}$ line, for $s=-1$ increasing $\sigma$ the spatial eigenvalues get closer to the imaginary axis. Nevertheless, for $s>\mu^{\prime}$ the situation remains qualitatively the same no matter how large is $\sigma$, as shown in Fig. 4(f). For $s<\mu^{\prime}$, as the range of interaction $\sigma$ increases one crosses the MI line, so that the HSS becomes modulationally unstable. Beyond the MI line the spatial dynamics is led by two imaginary doublets as shown in Fig. 4(i).

The spatial eigenvalues lie on a hyperbolalike curve for $\sigma$ high enough (cf. Fig. 4). Although the analytical solution is available [Eq. (23)], it does not yield a geometrically transparent picture of the locus of the curve on which the spatial eigenvalues lie. This behavior can be easily understood by neglecting the linear term versus the exponential one in (22),

$$
\exp \left(-\sigma^{2} u_{0} / 2\right)=1-\mu^{\prime} / s
$$

Using $u_{0}=-\left(q_{0}+i k_{0}\right)^{2}$ one gets

$$
\exp \left[\sigma^{2}\left(q_{0}^{2}-k_{0}^{2}\right) / 2+i \sigma^{2} q_{0} k_{0}\right]=1-\mu^{\prime} / s .
$$

From the modulus of (35) one has

$$
q_{0}^{2}-k_{0}^{2}=\frac{2}{\sigma^{2}} \ln \left|1-\frac{\mu^{\prime}}{s}\right|,
$$

which represents a hyperbola with eccentricity $\sqrt{2}$ in the complex plane. The right-hand side of (35) is real and therefore the phase $\sigma^{2} q_{0} k_{0}$ must be 0 or an integer multiple of $\pi$. For $\mu^{\prime} / s<1$,

$$
q_{0} k_{0}=\frac{2 n \pi}{\sigma^{2}}, \quad n \in \mathcal{Z} .
$$

Conversely, for $\mu^{\prime} / s>1$, the right-hand side of (35) is negative and

$$
q_{0} k_{0}=\frac{(2 n+1) \pi}{\sigma^{2}}, \quad n \in \mathcal{Z} .
$$

Equations (37) and (38) can be seen as a selection criterion, which has to be satisfied by a point on the hyperbola to be a spatial eigenvalue of the system. These hyperbolas are shown in Fig. 4. The approximation is meaningless for $\sigma \ll 1$ [cf. Figs. 4(a), 4(d), and 4(g)]. When $\sigma \geqslant 2$ it is clear that the above equation of the hyperbola provides a good approximation of the location of the spatial eigenvalues and the "selection criterion," in fact, gives eigenvalues that lie increasingly close to the real ones. Notice that the shape of the hyperbola depends on the sign of the logarithm in (36). For panels in the top and middle rows, $\left|1-\mu^{\prime} / s\right|>1$, while for the panels in the bottom row $\left|1-\mu^{\prime} / s\right|<1$. Thus, the hyperbola in the latter case has a conjugated shape as compared to the one in the panels of the top and middle rows.

Regarding temporal instabilities, for $s \sigma^{2}<-2$ the HSSs are modulationally unstable for $\mu^{\prime}>\mu_{\mathrm{MI}}^{\prime}$, as indicated by Eq. (27). However, it is necessary to take into account that a given value of $\mu^{\prime}$ corresponds to $\mu=\mu^{\prime}$ for the zero
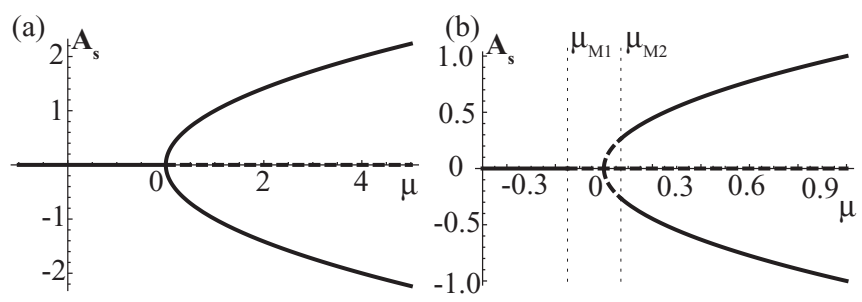

FIG. 6. Bifurcation diagram of the HSS of the nonlocal GLE with a Gaussian kernel and for $\sigma=2$ : (a) $s=0$ (local case); (b) $s=-1$. Stable solutions are shown by a solid line, while the unstable ones are shown by a dashed line.

HSS and to $\mu=-\mu^{\prime} / 2$ for the nonzero HSS [see Eq. (5)]. Therefore, the zero HSS becomes unstable due to MI at a value $\mu_{\mathrm{M} 1}=\mu_{\mathrm{MI}}^{\prime}<0$ and remains modulationally unstable for any $\mu>\mu_{\mathrm{M} 1}$. Similarly, the two nonzero symmetric HSSs are modulationally unstable for $0<\mu<\mu_{\mathrm{M} 2}$, where $\mu_{\mathrm{M} 2}=$ $-\mu_{\mathrm{MI}}^{\prime} / 2$. As a consequence in the interval $\left[\mu_{\mathrm{M} 1}, \mu_{\mathrm{M} 2}\right]$ there are no stable HSSs. For the parameters of Fig. 6(b), using Eq. (27) one has $\mu_{\mathrm{MI}}^{\prime}=-1+(1+\ln 2) / 2 \approx-0.153426$, from which it follows $\mu_{\mathrm{M} 1} \approx-0.153426$ and $\mu_{\mathrm{M} 2} \approx 0.076713$. Figure 7 shows the dependence of $\mu_{\mathrm{MI}}^{\prime}$ on $s$ for three different values of $\sigma$. In the limit of $\sigma \rightarrow \infty$ this dependence is given by the line $\mu^{\prime}=s$. The effect of a nonlocal nonlinear response in a MI is also discussed in Ref. [11,12].

Finally, we compare the results obtained here with an expansion up to the fourth moment, as discussed in Sec. III. Figure 2 shows in dotted lines the location of the BD and MI manifolds given by (18). The prediction given by the fourth moment expansion is quite good for the BD transition. The prediction for the MI manifold, while following the correct trend, becomes quite off as soon as one moves away from the QZ point, where $u_{c}=0$. As for the dependence of $s_{\mathrm{RDZ}}$ on the kernel width for a fixed $\mu^{\prime}$, one can use Eq. (21) with $\mathcal{M}_{2}=1$ and $\mathcal{M}_{4}=3$ (see Table I). As shown in Fig. 3 for the BD transition, the result given by fourth moment approximation (dashed line) is in good agreement with the exact one (solid line). In the case of the MI, the fourth order expansion does not work quite as well. In the limit of zero interaction range, it correctly predicts an asymptotic behavior $s_{\mathrm{RDZ}} \rightarrow-2 / \sigma^{2}$; however, in the limit of infinite interaction range, $\sigma \rightarrow \infty$, the prediction is $s_{\mathrm{RDZ}} \rightarrow 2 \mu^{\prime}$.

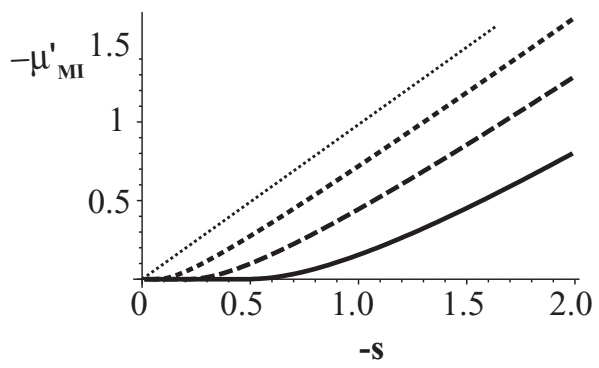

FIG. 7. Location of the MI in parameter space for the GLE with Gaussian kernel as a function of the nonlocal strength $s$ for different values of the interaction range: The solid, long-dashed, and shortdashed lines correspond, respectively, to $\sigma=2,3,5$, while the dotted line represents the $\sigma \rightarrow \infty$ limit. 


\section{THE MOD-EXPONENTIAL KERNEL}

We consider here the effect of a kernel whose profile decays exponentially in space on the tails of fronts starting (or ending) in an HSS of the GLE. In Fourier space the mod-exponential kernel is a Lorentzian (cf. Table I),

$$
\tilde{\hat{K}}(u)=\frac{1}{1+4 \sigma^{2} u},
$$

which in the complex plain has a singularity at $u=-1 /\left(4 \sigma^{2}\right)$. The dispersion relation obtained linearizing around the HSS is given by

$$
\tilde{\Gamma}(u)=\mu^{\prime}-u-s+\frac{s}{1+4 \sigma^{2} u} .
$$

The spatial eigenvalues are the zeros of $\tilde{\Gamma}(u)$. In this case, there are only four spatial eigenvalues $\lambda_{0}$ given by

$$
\begin{aligned}
u_{0} & =-\lambda_{0}^{2} \\
& =\frac{1}{2}\left[\mu^{\prime}-s-\frac{1}{4 \sigma^{2}} \pm \sqrt{\left(s-\mu^{\prime}+\frac{1}{4 \sigma^{2}}\right)^{2}+\frac{\mu^{\prime}}{\sigma^{2}}}\right] .
\end{aligned}
$$

MI and BD instabilities are located on the RDZ manifold of $\Gamma(u)$, which is given by $\tilde{\Gamma}\left(u_{c}\right)=\tilde{\Gamma}^{\prime}\left(u_{c}\right)=0$ :

$$
\begin{aligned}
& u_{c}=\frac{-1 \pm 2 \sigma \sqrt{-s}}{4 \sigma^{2}}, \\
& \mu^{\prime}=s+\frac{1}{4 \sigma^{2}}+2 u_{c} .
\end{aligned}
$$

MI and $\mathrm{BD}$ transitions require real $u_{c}$; thus, $\mathrm{MI}$ and $\mathrm{BD}$ transitions can only exist for $s<0$, namely for repulsive nonlocal interaction. Combining (42) and (43) to eliminate $u_{c}$, one obtains the RDZ manifold which in the $\left(\mu^{\prime}, s, \sigma\right)$ parameter space is the surface given by

$$
\mu_{\mathrm{RDZ}}^{\prime}=s-\frac{1}{4 \sigma^{2}} \pm \frac{\sqrt{-s}}{\sigma} .
$$

Setting $u_{c}=0$ in (42) and (43), one obtains the QZ codim-2 bifurcations (lines in the 3D parameter space). It turns out that there are two QZ lines. The first one takes place for finite $\sigma$ and is given by

$$
\mu_{\mathrm{QZ1}}^{\prime}=0, \quad s_{\mathrm{QZ} 1}=-\frac{1}{4 \sigma^{2}} .
$$

For the parameters of QZ1, $\tilde{\Gamma}^{\prime \prime}(0)=32 s_{\mathrm{QZ} 1} \sigma^{4}=-8 \sigma^{2}<0$; thus, in the notation of Part I, this is a $\mathrm{QZ}^{-}$point. The second QZ line is located at

$$
\sigma_{\mathrm{QZ} 2}=\infty, \quad \mu_{\mathrm{QZ} 2}^{\prime}=s .
$$

For the parameters of QZ2, $\tilde{\Gamma}^{\prime \prime}(0)=32 s \sigma_{\mathrm{QZ2}}^{4}=-\infty$; thus, this is also a $\mathrm{QZ}^{-}$point, albeit a particular one since the second derivative is infinite. As a consequence, the parabola described by the RDZ close to QZ2 is infinitely narrow, and the $\mathrm{BD}$ and MI lines unfold from QZ2 practically tangentially.

In the part of the RDZ manifold that starts from the side $s \sigma^{2}<-1 / 4$ of QZ1 and the side $s<\mu^{\prime}$ of QZ2, $u_{c}>0$, it thus corresponds to a MI bifurcation. On the other part $u_{c}<0$, thus corresponds to a BD.

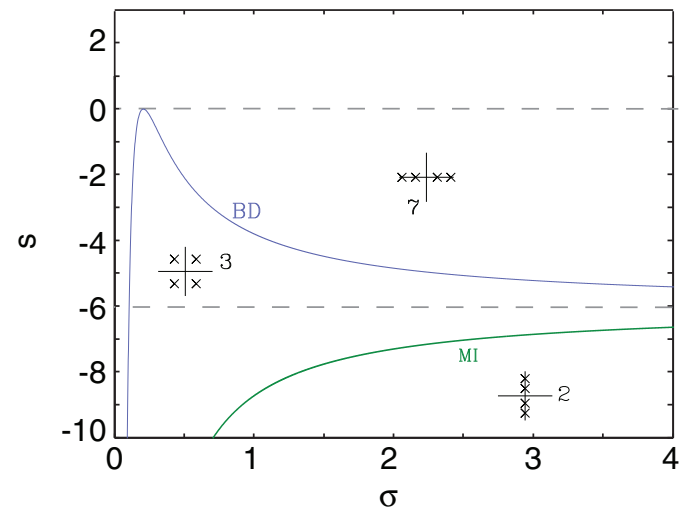

FIG. 8. (Color online) Boundaries in the $(\sigma, s)$ plane separating the regions of monotonic and oscillatory tails in the GLE with a modexponential kernel for $\mu^{\prime}=-6$ obtained from (47). The horizontal dashed line at $s=-6$ shows the asymptotic limit of MI and BD lines for $\sigma \rightarrow \infty$.

Cusp or 3DZ codim-2 points cannot exist for the GLE with a mod-exponential kernel since they require at least six spatial eigenvalues. The crossover manifold does not exist either in this case.

We now address the effect of the mod-exponential kernel for a given value of $\mu^{\prime}$. It is convenient to rewrite (44) as

$$
s_{\mathrm{RDZ}}=\mu^{\prime}-\frac{1}{4 \sigma^{2}} \pm \frac{1}{\sigma} \sqrt{-\mu^{\prime}} .
$$

The + and - signs correspond to the BD and MI manifolds, respectively. The MI and BD transitions are shown in Fig. 8 for the nonzero HSSs for $\mu^{\prime}=-6$ corresponding to $\mu=3$. LSs are found in the parameter region bounded by the BD and MI curves. The existence of QZ2 leads to a significant difference with the Gaussian kernel (cf. Fig. 3). Increasing $\sigma$, the BD and MI lines tend asymptotically one to the other and meet at the QZ2. As a consequence the region of LSs narrows as $\sigma$ increases. The curve $s_{\mathrm{BD}}$ has a maximum at $\sigma^{\dagger}=1 /(2 \sqrt{2 \mu})$ (i.e., at $\sigma=1 / \sqrt{24}=0.204124$ in Fig. 8), where it reaches $s=0$, the maximum value of $s$ for which the RDZ manifold exists. For $\mu \rightarrow 0$ the BD and MI curves approach each other and meet at QZ1.

Considering the MI and comparing with the local case shown in Fig. 6(a), one also finds that there is a finite range of values of $\mu \in\left[\mu_{\mathrm{M} 1}, \mu_{\mathrm{M} 2}\right]$ around the pitchfork bifurcation, $\mu=0$, that are modulationally unstable. For the parameters of Fig. 6(b), $\sigma=2$ and $s=-1<-1 /\left(4 \sigma^{2}\right), \mu_{\mathrm{M} 1}=-9 / 16$ and $\mu_{\mathrm{M} 2}=-\mu_{\mathrm{M} 1} / 2=9 / 32$.

Written in terms of $u$ the mod-exponential kernel in Fourier space (39) has a pole of order 1 at $u=-1 /\left(4 \sigma^{2}\right)$. As a consequence a moment expansion around $u=0$ such as the one discussed in Sec. III A of Part I will converge only for $|u|<1 /\left(4 \sigma^{2}\right)$, and therefore it will be of limited use. A truncation up to $M_{4}$ identifies the spatial behavior around the first QZ, in particular the existence of spatial tails, and thus of LSs, for $s<0$, but as $\sigma$ is increased, the predictions from moment expansion are quite off. This kernel can be considered as the simplest with singularities, and no further approximations can be obtained from a Laurent expansion (Sec. III B of Part I) since $1 /\left(1+4 \sigma^{2} u\right)$ is already the first and 
only term of that expansion. Still, proceeding as in Sec. III B of Part I, one can obtain an exact transformation for the nonlocal interaction term. Using (39) the nonlocal interaction can be written as

$$
\hat{F}(k, \sigma)=\frac{1}{1+4 \sigma k^{2}} \hat{A}(k),
$$

where $\hat{A}(k)$ is the Fourier transform of $A(x)$. In real space this leads to

$$
\left(1-4 \sigma \partial_{x x}\right) F(x, \sigma)=A(x),
$$

which is an ordinary differential equation. Therefore, the GLE with a mod-exponential nonlocal kernel can be written as a partial differential equation coupled to an ordinary differential equation,

$$
\begin{array}{r}
\partial_{t} A=(\mu-s) A-A^{3}+\partial_{x x} A+s F(x, \sigma), \\
\partial_{x x} F(x, \sigma)=\frac{1}{4 \sigma}[F(x, \sigma)-A(x)],
\end{array}
$$

where we have used that $M_{0}=1$. This treatment of the modexponential kernel was introduced in Ref. [34] and used also by other authors $[10,17]$.

\section{THE MEXICAN-HAT KERNEL}

In this section, we discuss in detail the effects of a spatially nonlocal kernel that is not everywhere positively defined. More precisely the kernel we consider consists of two Gaussians and has an extra parameter $b>0$ (cf. Table I) that regulates the spatial extension of the negative sector of the kernel. The total area of this kernel is given by $M_{0}=2(1-b)$ (cf. Table I), and for $b>1, M_{0}<0$. For $s>0$ one has short-range attraction (activation) and medium to long-range repulsion (inhibition). In this case, activation dominates globally for $b<1$, while otherwise overall inhibition is stronger than activation. For $s<0$ one has inhibition in the short range and activation in the medium to long range and globally inhibition dominates for $b<1$ while activation does otherwise.

The dispersion relation for this kernel is [cf. Eq. (7) and Table I],

$$
\tilde{\Gamma}(u)=\mu^{\prime}-2 s(1-b)-u+2 s\left(1-b+b \sigma^{2} u\right) e^{-\sigma^{2} u / 2},
$$

and setting $\tilde{\Gamma}(u)=0$ does not lead to a closed expression for the spatial eigenvalues, which have to be obtained numerically for this kernel. The RDZ manifold is given by $\tilde{\Gamma}\left(u_{c}\right)=\tilde{\Gamma}^{\prime}\left(u_{c}\right)=0$ :

$$
\begin{gathered}
\frac{1}{s \sigma^{2}}=\left(-1+3 b-b \sigma^{2} u_{c}\right) e^{-\sigma^{2} u_{c} / 2}, \\
\mu^{\prime}=2 s-2 s b+\frac{2}{\sigma^{2}}+u_{c}-4 s b e^{-\sigma^{2} u_{c} / 2} .
\end{gathered}
$$

Since now the parameter space $(\mu, s, \sigma, b)$ is $4 \mathrm{D}$, the RDZ manifold is a 3D hypersurface. Equation (52) can be solved analytically for $u_{c}$,

$$
u_{c, l}=\frac{1}{\sigma^{2}}\left[3-\frac{1}{b}-2 W_{l}(\chi)\right]
$$

where $l=0,-1$ are the indices of the two real branches of the Lambert $W$ function (see the Appendix) and

$$
\chi=\chi(b, s, \sigma)=\frac{1}{2 b s \sigma^{2}} \exp \left(\frac{3 b-1}{2 b}\right) .
$$

Substituting (54) into (53), one gets

$$
\mu_{\mathrm{RDZ}, l}^{\prime}=2 s(1-b)+\frac{5}{\sigma^{2}}-\frac{1}{b \sigma^{2}}-\frac{2 W_{l}(\chi)}{\sigma^{2}}-\frac{2}{\sigma^{2} W_{l}(\chi)} .
$$

For $-1 / e<\chi<0$ the RDZ manifold has two branches which we label $l=0$ and $l=-1$ as the indices of the $W$ function (see the Appendix). For $\chi>0$ the RDZ manifold has a single branch given by $l=0$. For $\chi<-1 / e, W$ does not take real values; thus, there is no RDZ manifold. The asymptotic behavior of the RDZ branches for large $s$ is given by

$$
\begin{gathered}
\mu_{\mathrm{RDZ}, 0}^{\prime}=2 s\left[1-b-2 b \exp \left(\frac{1-3 b}{2 b}\right)\right] \\
+\frac{3 b-1}{b \sigma^{2}}+\mathcal{O}\left(s^{-1}\right), \\
\mu_{\mathrm{RDZ},-1}^{\prime}=2 s(1-b)+\mathcal{O}[\ln (s)] .
\end{gathered}
$$

The part of the RDZ manifold with $u_{c}<0$ corresponds to a $\mathrm{BD}$ transition while the part with $u_{c}>0$ corresponds to a $\mathrm{HH}$ bifurcation. Note that the $\mathrm{BD}$ and $\mathrm{HH}$ parts of the RDZ manifold are not directly related to the index $l$ of $\mu_{\mathrm{RDZ}, l}^{\prime}$. Instead, as discussed below, $\mu_{\mathrm{RDZ}, 0}^{\prime}$ have both $\mathrm{BD}$ and $\mathrm{HH}$ parts and the same applies to $\mu_{\mathrm{RDZ},-1}^{\prime}$. For the moment, we distinguish the part of the BD and MI manifolds in which the second derivative of the dispersion relation $\Gamma(u)$, given by

$$
\tilde{\Gamma}^{\prime \prime}(u)=\frac{s \sigma^{4}}{2}\left(1-5 b+b \sigma^{2} u\right) e^{-\sigma^{2} u / 2},
$$

is positive from that where is negative. The part of the RDZ with $\tilde{\Gamma}^{\prime \prime}\left(u_{c}\right)>0$ corresponds to a local minimum of the dispersion relation crossing zero and in this section it will be referred to as $\mathrm{HH}^{+}$or $\mathrm{BD}^{+}$, while the part with $\tilde{\Gamma}^{\prime \prime}\left(u_{c}\right)<0$ corresponds to a local maximum of the dispersion relation crossing zero and will be referred to as as $\mathrm{HH}^{-}$or $\mathrm{BD}^{-}$. If the local maximum signaled by the $\mathrm{HH}^{-}$turns out to be the global maximum, then it corresponds to a MI of the HSS and, as in previous sections, it is referred to as MI.

Setting $u_{c}=0$ in Eqs. (52) and (53), one finds the QZ manifold, which in the $(\mu, s, \sigma, b)$ parameter space is a $2 \mathrm{D}$ surface given by

$$
\mu_{\mathrm{QZ}}^{\prime}=0, \quad s_{\mathrm{QZ}}=\frac{1}{\sigma^{2}(3 b-1)} .
$$

$s_{\mathrm{QZ}}$ has a divergence at $b=1 / 3$, and, as a consequence, the QZ manifold splits into two parts. For $b<1 / 3, s_{\mathrm{QZ}}<0$, as shown in Fig. 9(a), while for $b>1 / 3, s_{\mathrm{QZ}}>0$, as shown in Fig. 9(d). This is a clear distinction with the previous two kernels for which $s_{\text {QZ }}$ was always negative.

As discussed in Part I there are two kinds of QZ points depending on the sign of $\Gamma^{\prime \prime}(u)$ at the QZ point. From (59) we 

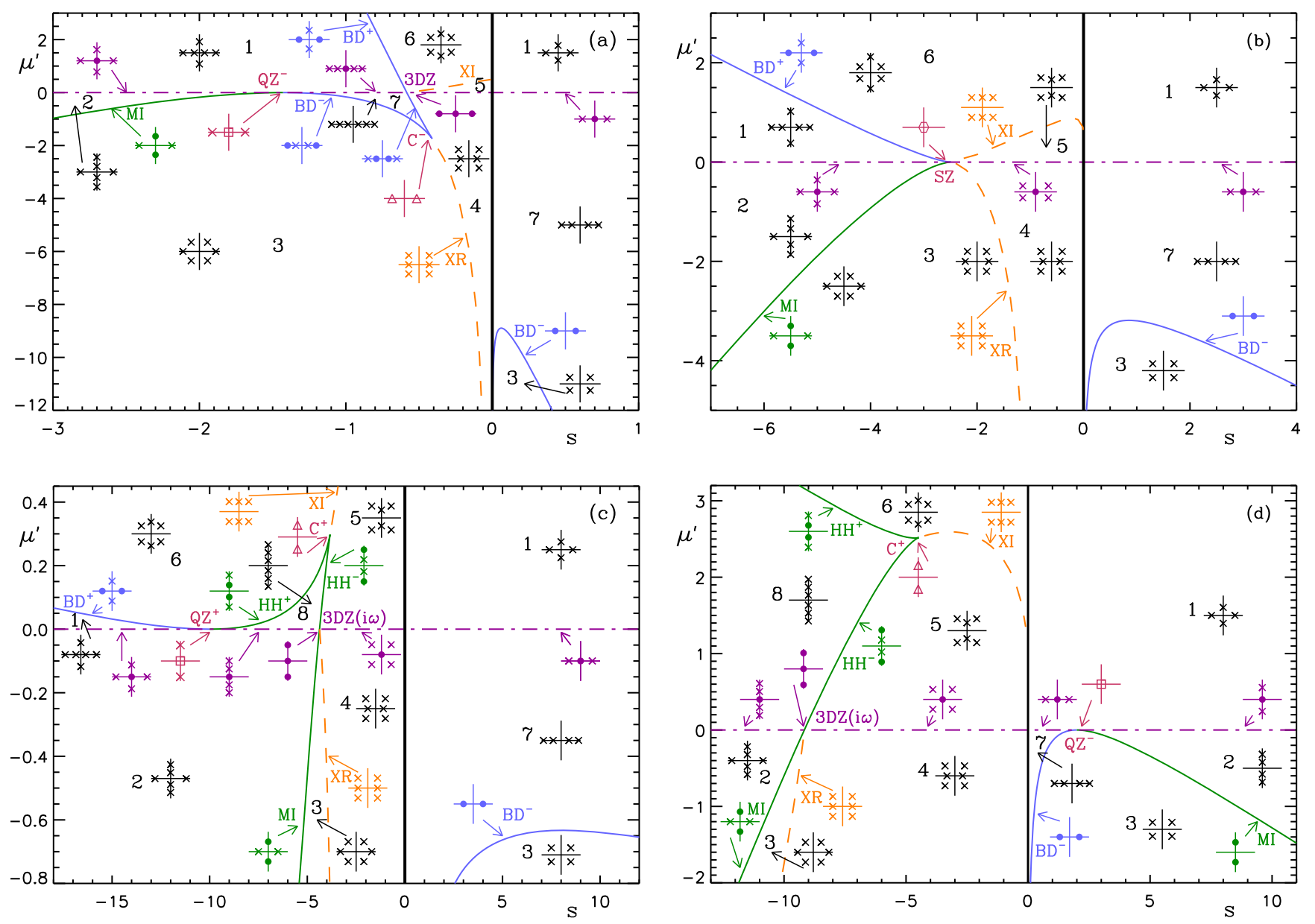

FIG. 9. (Color online) Boundaries in the $\left(\mu^{\prime}, s\right)$ parameter space at which the leading spatial eigenvalues of the GLE with a Mexican-hat nonlocal kernel exhibit different transitions for $\sigma=1$ and (a) $b=0.1$, (b) $b=b_{\mathrm{SZ}}=0.2$, (c) $b=0.3$, and (d) $b=0.5$. Sketches indicate the position of the zeros of $\Gamma_{s}(\lambda)$ in the $[\operatorname{Re}(\lambda), \operatorname{Im}(\lambda)]$ plane $(\times$, signal simple zeros; $\bullet$, double zeros; $\triangle$, triple zeros; $\square$, QZs; and $\square$, sextuple zeros).

have

$$
\tilde{\Gamma}_{\mathrm{QZ}}^{\prime \prime}(0)=\frac{s_{\mathrm{QZ}} \sigma^{4}}{2}(1-5 b)=-\frac{\sigma^{2}}{2} \frac{1-5 b}{1-3 b}
$$

For $\tilde{\Gamma}_{\mathrm{QZ}}^{\prime \prime}(0)<0$ the $\mathrm{QZ}$ is a $\mathrm{QZ}^{-}$unfolding a $\mathrm{BD}^{-}$and a MI manifolds towards $\mu^{\prime}<0$ [see Figs. 9(a) or 9(d)], while for $\tilde{\Gamma}_{\mathrm{QZ}}^{\prime \prime}(0)>0$ one has a $\mathrm{QZ}^{+}$unfolding a $\mathrm{BD}^{+}$and a $\mathrm{HH}^{+}$ manifolds towards $\mu^{\prime}>0$ [see Fig. 9(c)].

At difference with the previous kernels, the GLE with a Mexican-hat nonlocal kernel exhibits a codim-2 cusp manifold. Setting $\tilde{\Gamma}^{\prime \prime}\left(u_{\text {cusp }}\right)=0$, one has

$$
u_{\text {cusp }}=\frac{1}{\sigma^{2}}\left(5-\frac{1}{b}\right) \text {. }
$$

From $\tilde{\Gamma}^{\prime}\left(u_{\text {cusp }}\right)=0$ one obtains

$$
s_{\text {cusp }}=-\frac{1}{2 b \sigma^{2}} \exp \left(\frac{5}{2}-\frac{1}{2 b}\right) .
$$

Finally, setting $\tilde{\Gamma}\left(u_{\text {cusp }}\right)=0$ and using (63), one arrives at

$$
\mu_{\text {cusp }}^{\prime}=\frac{1}{\sigma^{2}}\left[9-\frac{1}{b}+\left(1-\frac{1}{b}\right) \exp \left(\frac{5}{2}-\frac{1}{2 b}\right)\right] \text {. }
$$

From Eqs. (62) and (54), one finds that at the cusp $W_{l}\left(\chi_{\text {cusp }}\right)=-1$, which is the branching point of the Lambert $W$ function where the two real branches $W_{0}$ and $W_{-1}$ originate (cf. the Appendix). These two $W$ branches associated with the two branches of the $\mu_{\mathrm{RDZ}}^{\prime}$ manifold which in parameter space emerge from the cusp one tangent to the other [see for example point $C^{-}$in Fig. 9(a)]. Since $W_{-1}(\chi)<-1$ for any value of $\chi$ (cf. the Appendix), on the branch $\mu_{\mathrm{RDZ},-1}^{\prime}$, $u_{c,-1}>u_{\text {cusp }}$ and, since (59) can be written as $\tilde{\Gamma}^{\prime \prime}(u)=$ $\left(s b \sigma^{6} / 2\right)\left(u-u_{\text {cusp }}\right) \exp \left(-\sigma^{2} u / 2\right)$, the sign of $\tilde{\Gamma}^{\prime \prime}\left(u_{c,-1}\right)$ is that of $s b$. On the contrary, on the branch $\mu_{\mathrm{RDZ}, 0}^{\prime}, u_{c, 0}<u_{\text {cusp }}$ and $\tilde{\Gamma}^{\prime \prime}\left(u_{c, 0}\right)>0$ for $s b<0$, while $\tilde{\Gamma}^{\prime \prime}\left(u_{c}, 0\right)<0$ for $s b>0$. Notice that (63) implies $s_{\text {cusp }} b<0$, so close to the cusp $s b<0$.

For $u_{\text {cusp }}<0$ the cusp is, in the notation of Part $\mathrm{I}$, a $\mathrm{C}^{-}$ unfolding a $\mathrm{BD}^{+}$manifold and a $\mathrm{BD}^{-}$manifold, as is the case of Fig. 9(a). The $\mathrm{BD}^{+}$corresponds to $\mu_{\mathrm{RDZ}, 0}^{\prime}$ and the $\mathrm{BD}^{-}$to $\mu_{\mathrm{RDZ},-1}^{\prime}$. Moving away from the cusp along the $\mathrm{BD}^{-}$line, $u_{c,-1}$ increases and eventually it reaches zero at the $\mathrm{QZ}^{-}$, where the $\mathrm{BD}^{-}$becomes a MI. The $\mathrm{C}^{-}$cusp also unfolds a crossover manifold at which a real doublet and a complex quartet are located at the same distance from the imaginary axis which we label as XR. In parameter space XR can be seen as the 
natural continuation of the two BD manifolds that end one tangent to the other at the cusp [see Fig. 9(a)].

For $u_{\text {cusp }}>0$ the cusp is a $\mathrm{C}^{+}$unfolding a $\mathrm{HH}^{+}$and a $\mathrm{HH}^{-}$ which correspond to $\mu_{\mathrm{RDZ}, 0}^{\prime}$ and to $\mu_{\mathrm{RDZ},-1}^{\prime}$, respectively [see Fig. 9(c)]. Moving along the $\mathrm{HH}^{+}$manifold away from the cusp, $u_{c, 0}$ decreases and eventually it reaches zero at the $\mathrm{QZ}^{+}$ point where the $\mathrm{HH}^{+}$becomes a $\mathrm{BD}^{+}$. Also unfolding from $\mathrm{C}^{+}$there is a crossover manifold (labeled as $\mathrm{XI}$ ) at which a imaginary doublet and a complex quartet are located at the same distance from the real axis. Similarly as before, in parameter space XI can be seen as the continuation of the two $\mathrm{HH}$ manifolds ending at the cusp.

The GLE with a Mexican-hat kernel also has a 3DZ codim-2 point and a 3DZ $(i \omega)$ codim-2 point. As discussed in Part I, at these points a simple zero at the origin $\tilde{\Gamma}(0)=0$ and a RDZ at finite distance, $\tilde{\Gamma}\left(u_{c}\right)=\tilde{\Gamma}^{\prime}\left(u_{c}\right)=0$, take place simultaneously. Since $\tilde{\Gamma}(0)=0$ implies $\mu^{\prime}=0$, the $3 \mathrm{DZ}$ and $3 \mathrm{DZ}(i \omega)$ can be obtained setting $\mu_{\mathrm{RDZ}}^{\prime}=0$ in Eq. (56) and looking for solutions with nonzero $u_{c}$. Thus, within the $\mu^{\prime}=0$ hyperplane the location of the $3 \mathrm{DZ}$ and $3 \mathrm{DZ}(i \omega)$ is given by the implicit equation

$$
0=2 s \sigma^{2}(1-b)+5-\frac{1}{b}-2 W_{l}(\chi)-\frac{2}{W_{l}(\chi)} .
$$

The 3DZ $(i \omega)$ point is located on the $\mathrm{HH}^{-}$manifold that unfolds from the $\mathrm{C}^{+}$cusp [see Figs. 9(c) and 9(d)] and has a significant effect on it. At this point $\mathrm{HH}^{-}$, which at the cusp is a local maximum of the dispersion relation crossing zero, becomes a global maximum. Thus, the $\mathrm{HH}$ bifurcation becomes a MI [see Fig. 9 of Part I]. Similarly, the 3DZ point is located on the $\mathrm{BD}^{+}$manifold that unfolds from the $\mathrm{C}^{-}$cusp [see Fig. 9(a)].

The sextuple zero point, which organizes the overall scenario, takes place when $u_{\text {cusp }}=0$, which in the $(\mu, s, \sigma, b)$ parameter space is the line

$$
b_{\mathrm{SZ}}=\frac{1}{5}, \quad s_{\mathrm{SZ}}=-\frac{5}{2 \sigma^{2}}, \quad \mu_{\mathrm{SZ}}=0 .
$$

The parameter space portrait for the GLE with a Mexicanhat kernel is as follows. For $b=b_{\mathrm{SZ}}$ [Fig. 9(b)] the SZ unfolds a $\mathrm{BD}^{+}\left(\mu_{\mathrm{RDZ}, 0}^{\prime}\right)$ manifold, a $\mathrm{MI}\left(\mu_{\mathrm{RDZ},-1}^{\prime}\right)$ manifold, an XR manifold, and an XI manifold in a way similar to that described in Part I for the sixth-order dispersion relation in $\lambda$. For negative and large $s$ the asymptotic behavior of $\mathrm{BD}^{+}$is given by Eqs. (57) and that of MI by (58). The part for $s<0$ of Fig. 9(b) can be directly compared with Fig. 2(b) of Part I. We refer to Part I for a detailed explanation of all the regions surrounding the SZ as well as the transitions between them. The regions relevant for the existence of stable LSs are region 3, where the spatial dynamics is led by a complex quartet and the part of region 4 close to the crossover $X R$, where the spatial dynamics results from the combination of a real doublet and a complex quartet. The $s=0$ line corresponds to the GLE with local coupling with two spatial eigenvalues which are real $\mu^{\prime}<0$ and imaginary for $\mu^{\prime}>0$. For $s>0$ and $\mu^{\prime}<0$ there is a BD ${ }^{-}$ line given by $\mu_{\mathrm{RDZ}, 0}^{\prime}$ which separates region 3 led by a complex quartet from region 7 led by two real doublets, in which fronts are monotonic. For large positive $s$ the $\mathrm{BD}^{-}$line follows the asymptotic behavior (57). Thus, the $\mathrm{BD}^{+}$line for $s<0$ and the $\mathrm{BD}^{-}$line for $s>0$ have the same oblique asymptote. For $s \rightarrow 0$ the $\mathrm{BD}^{-}$line goes to $-\infty$. When crossing $\mu^{\prime}=0$ from region 7 into region 1 , the components of the doublet closer to the origin collide, leading to an imaginary doublet. This is a Hamiltonian-pitchfork bifurcation as described in Part I.

For $b<b_{\mathrm{SZ}}$ the SZ unfolds a $\mathrm{QZ}^{-}$within the $\mu^{\prime}=0$ hyperplane and a cusp located at $\mu^{\prime}<0$, as shown in Fig. 9(a) for $b=0.1$. From Eq. (62), for $b<b_{\mathrm{SZ}}, u_{\text {cusp }}<0$; thus, this is a $\mathrm{C}^{-}$cusp unfolding two BD manifolds and a crossover XR. The $\mathrm{BD}^{-}$manifold (given by $\mu_{\mathrm{RDZ},-1}^{\prime}$ ) connects $\mathrm{C}^{-}$with $\mathrm{QZ}^{-}$, where it becomes an MI. The $\mathrm{BD}^{+}$manifold (given by $\mu_{\mathrm{RDZ}, 0}^{\prime}$ ) connects $\mathrm{C}^{-}$with the $3 \mathrm{DZ}$ located in the $\mu^{\prime}=0$ hyperplane. For large negative $s$ the asymptotic behavior of both manifolds is given by Eqs. (57) and (58). The overall picture shown in the part of Fig. 9(a) corresponding to $s<0$ has the same structure as Fig. 2(a) of Part I, to which we refer for a discussion. As for $s>0$ the regions are the same as in Fig. 9(b) but the $\mathrm{BD}^{-}$ line given by $\mu_{\mathrm{RDZ}, 0}^{\prime}$ has moved down to more negative values of $\mu^{\prime}$ [notice the different vertical scale in Figs. 9(a) and 9(b)] and region 3 has narrowed. As a consequence one has a large parameter region for $s<0$, where LSs may be formed, which includes region 3 unfolding from $\mathrm{QZ}^{-}$and the part of region 4 close to XR while for $s>0$ there is a narrow region 3 located at small $s$ and reachable only for strongly negative values of $\mu^{\prime}$.

If $b$ is further decreased, the $C^{-}$cusp moves towards smaller values of $s$ and more negative values for $\mu^{\prime}$. At the same time the $\mathrm{BD}^{+}$line born at the right of the cusp becomes more vertical. The $\mathrm{BD}^{-}$line at $s>0$ is located further down and region 3 keeps narrowing. In the limit $b \rightarrow 0^{+}$the cusp goes to $s_{\text {cusp }} \rightarrow 0^{-}$and $\mu_{\text {cusp }}^{\prime} \rightarrow-\infty$, while the $\mathrm{BD}^{-}$line located in the $s>0$ semiplane goes also to $-\infty$. The result is that one recovers the parameter space diagram obtained for the Gaussian kernel (Fig. 2).

For $b>b_{\mathrm{SZ}}$ the SZ unfolds a $\mathrm{QZ}^{+}$and a $3 \mathrm{DZ}(i \omega)$ manifolds located within the $\mu^{\prime}=0$ hyperplane and a cusp located at $\mu^{\prime}>0$ as shown in Fig. 9(c) for $b=0.3$. From Eq. (62), for $b_{\mathrm{SZ}}, u_{\text {cusp }}>0$; thus, this is a $\mathrm{C}^{+}$cusp unfolding two $\mathrm{HH}$ manifolds and a crossover XI. The $\mathrm{HH}^{+}$manifold (given by $\mu_{\mathrm{RDZ}, 0}^{\prime}$ ) connects $\mathrm{C}^{+}$with $\mathrm{QZ}^{+}$, where it becomes a $\mathrm{BD}^{+}$. The $\mathrm{HH}^{-}$manifold (given by $\mu_{\mathrm{RDZ},-1}^{\prime}$ ) connects $\mathrm{C}^{+}$with the 3DZ $(i \omega)$. Globally, the part for $s<0$ of Fig. 9(c) has the same regions and transitions as those obtained for $b>0$ for the sixth-order dispersion relation considered in Part I [Fig. 2(c)]. For $s<0$ region 3 now unfolds from the 3DZ $(i \omega)$ point and has a sharp-pointed shape and thus is narrower than in Figs. 9(a) and 9(b). For $s>0$ the regions are the same as in Fig. 9(b) but the $\mathrm{BD}^{-}$line given by $\mu_{\mathrm{RDZ}, 0}^{\prime}$ has moved up and region 3 has widened significantly. Still, in order to reach region 3 for $s>0$ it is necessary that $\mu^{\prime}$ is not too close to zero.

If $b$ is further increased, the $\mathrm{QZ}^{+}$point moves towards more negative values for $s$. For $b \rightarrow 1 / 3^{-}, s_{\mathrm{QZ}} \rightarrow-\infty$ [cf. Eq. (60)]. Also, the slope and the ordinate at the origin of the oblique asymptote of $\mu_{\mathrm{RDZ}, 0}^{\prime}$ tend to zero as $b \rightarrow 1 / 3$, as can be seen from Eq. (57). For $b>1 / 3$, the QZ point is located at $s_{\mathrm{QZ}}>0$, starting from $s_{\mathrm{QZ}}=\infty$ at $b=1 / 3^{+}$ and monotonically approaching $s_{\mathrm{QZ}}=0$ as $b$ increases. The $\mathrm{QZ}$ is, in fact, a $\mathrm{QZ}^{-}$point since for $b>1 / 3, \tilde{\Gamma}_{\mathrm{QZ}}^{\prime \prime}(0)<0$ [see Fig. 9(d) for $b=1 / 2$ ]. The $\mathrm{QZ}^{-}$unfolds a $\mathrm{BD}^{-}$and a MI manifolds given by $\mu_{\mathrm{RDZ}, 0}^{\prime}$. The cusp $\mathrm{C}^{+}$is still present in the $\mu^{\prime}>0, s<0$ quadrant, unfolding a $\mathrm{HH}^{+}$line which asymptotically connects with the MI line for $s>0$, since both 
are part of $\mu_{\mathrm{RDZ}, 0}^{\prime}$. As before, the $\mathrm{HH}^{-}$unfolding from $\mathrm{C}^{+}$ goes to the $3 \mathrm{DZ}(i \omega)$ point, where it becomes an MI. Now one has region 3 reaching all the way up to $\mu^{\prime}=0$ for both $s<0$ and $s>0$, and, in fact, region 3 is much larger for $s>0$ than for $s<0$. From a physical point of view this can be understood by noticing that for $b>1 / 3$ all the moments of the Mexican-hat kernel, except $M_{0}$ are negative. Thus, while the overall area of the kernel is positive and for $s>0$ the nonlocal interaction can be considered as globally attractive, at medium and long distances the nonlocal interaction is repulsive. This compensates the local attractive interaction leading to oscillations in the front profile.

Increasing $b$ in the half plane $s>0$, the $\mathrm{QZ}^{-}$approaches zero but there are no qualitative changes. In the half plane $s<0$ the slope of the $\mathrm{HH}^{-}$that unfolds from $\mathrm{C}^{+}$given by $\mu_{\mathrm{RDZ},-1}^{\prime}$ decreases [e.g., Eq. (58)] and the 3DZ $(i \omega)$ point moves towards more negative values of $s$. For $b=1$ the $3 \mathrm{DZ}(i \omega)$ point is located at $s \rightarrow-\infty$. For $b>1$ the $\mathrm{HH}^{-}$does not cross $\mu^{\prime}=0$ and there is no $3 \mathrm{DZ}(i \omega)$ point and, thus, no crossover XR nor region 3 for $s<0$. The result is that for $b>1$ only region 3 unfolded by $\mathrm{QZ}^{-}$located at $s>0$ remains as parameter regions where stable LSs can be formed.

We now focus on the effect of the Mexican-hat nonlocal kernel for given parameter values of the local GLE, that is for a given $\mu^{\prime}$. In what follows we take $b=1 / 2$ [Fig. 9(d)] so that the total area of the kernel is $M_{0}=1$, as in the kernels considered in the previous sections.

For this kernel a closed formula for $s_{\mathrm{RDZ}}\left(\mu^{\prime}, \sigma\right)$ is not available, but, nevertheless, it can be found semianalytically by replacing (54) with (53) with $b=1 / 2$. The result for $\mu^{\prime}=-6$ is shown in Fig. 10. As discussed above, the result is that one finds two sections of the RDZ manifold for $s>0, \mathrm{BD}^{-}$and $\mathrm{MI}$, unfolding from $\mathrm{QZ}^{-}$, while for $s<0$ one has a $\mathrm{MI}$ and a crossover XR unfolding from 3DZ $(i \omega)$ [see also Fig. 9(d)].

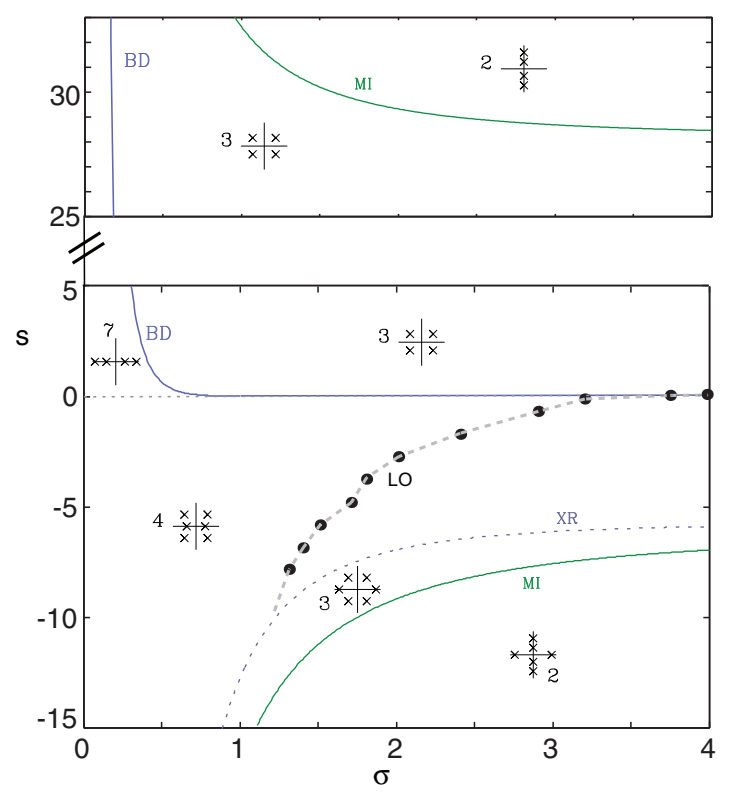

FIG. 10. (Color online) Boundaries in the $(\sigma, s)$ plane separating the regions of monotonic and oscillatory tails when using a Mexicanhat shaped kernel in the nonlocal GLE. $\mu^{\prime}=-6$.
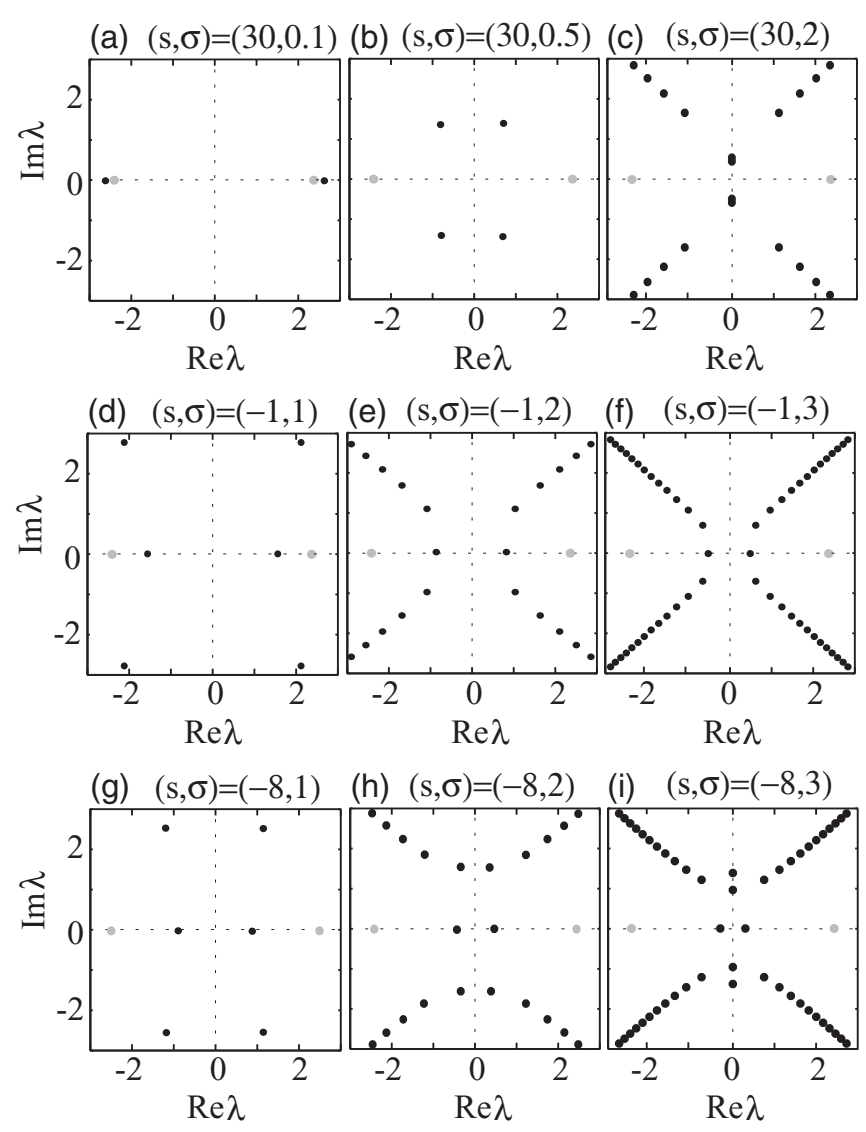

FIG. 11. Location of the first spatial eigenvalues for the GLE with a Mexican-hat nonlocal kernel in the complex $\lambda$ plane (shown as black dots) for $b=1 / 2$ and $\mu^{\prime}=-6$. The top row corresponds to $s=30$ with (a) $\sigma=0.1$, (b) $\sigma=0.5$, and (c) $\sigma=2$. The middle row corresponds to $s=-1$ with (d) $\sigma=1$, (e) $\sigma=2$, and (f) $\sigma=3$. The bottom row corresponds to $s=-8$ with (g) $\sigma=1$, (h) $\sigma=2$, and (i) $\sigma=3$. For comparison the two gray dots show the location of the eigenvalues for the local GLE $(s=0)$. In all the cases the number of spatial eigenvalues is infinite: The plot just presents the region around the origin in the complex plane.

Figure 11 shows the location in the complex $\lambda$ plane of the first few spatial eigenvalues for different values of $s$ and $\sigma$. The first row corresponds to $s=30$. For $\sigma$ small the spatial dynamics is led by a real doublet located close to the real doublet of the GLE with local coupling [Fig. 11(a)]. There is another real doublet located outside the region plotted in Fig. 11(a). Increasing $\sigma$ the second real doublet gets closer to the origin and collides with the first pair in a BD transition leading to a complex quartet [Fig. 11(b)] within parameter region 3 , where front tails have a oscillatory profile (see Fig. 10). Further increasing $\sigma$ leads to a collision of the components of the complex quartet on the imaginary axis (MI transition), which results in two imaginary doublets [Fig. 11(c)] within parameter region 2 .

For $b=1 / 2$ and $s<0$ the one finds only one real doublet in the spatial spectrum. For $s$ not too negative this real doublet leads the spatial dynamics for any value of $\sigma$ as shown in the second row of Fig. 11, which corresponds to $s=-1$. The three panels of this row are within parameter region 4 (see Fig. 10). 
For a large negative $s$ the real doublet leads the dynamics only for small $\sigma$ [see the eigenvalues plotted in Fig. 11(g) for parameters within region 4 of Fig. 10]. Increasing $\sigma$ one encounters the crossover XR after which there is a complex quartet located closer to the imaginary axis than the real doublet [Fig. 11(h)] and therefore one enters in the parameter region 3 unfolding from the $3 \mathrm{DZ}(i \omega)$ point. For larger values of $\sigma$ there is a MI transition at which the components of the complex quartet collide on the imaginary axis. After this the spatial dynamics is led by two imaginary pairs as shown in Fig. 11(i) for parameters within region 2.

As discussed in Part I in the part of region 4 located near the crossover XR, the real part of the complex quartet is close to that of the real doublet and the spatial dynamics results, in fact, from the combination of the real doublet and the complex quartet. Thus, although asymptotically the front tail is monotonic, closer to the front core the complex quartet manifests, introducing oscillations, whose role in the fronts interaction can lead to the existence of stable LSs. At difference with the case where the leading eigenvalues are complex, and the front tail has oscillations asymptotically, here the front shows oscillations only close to the core, and thus the locking of two fronts can only occur at short distances.

To determine the part of region 4 where this occurs we consider the full evolution equation (3) with the Mexican-hat nonlocal kernel. We set the initial condition such that there are two fronts connecting the two equivalent HSSs (kink and antiking) and look at the velocity at which the two fronts approach each other. In Ref. [1] (cf. Fig. 1) it was shown that the interaction of two monotonic fronts (in systems with two equivalent states) decays exponentially (both for local and spatially nonlocal interactions where the kernel decays faster than exponentially). In the part of region 4 where the fronts are no longer monotonic, the envelope of the interaction of two oscillatory fronts still decays exponentially, but at some particular distances the fronts pin and the relative velocity drops to zero. Figure 12 shows the dependence of the relative velocity $v(d)$ on the distance between the fronts $d$ (the width of the kink-antiking structure) for different values of the interaction range $\sigma$.

One can see that when the range of nonlocal interaction vanishes $(\sigma=0)$ the logarithm of the relative velocity grows linearly when decreasing $d$. Switching on the nonlocal inter-

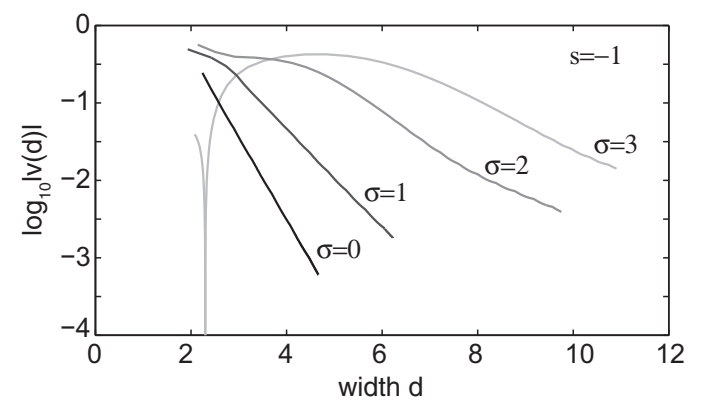

FIG. 12. Velocity at which two fronts connecting the two equivalent HSSs approach each other for the GLE equation with a Mexican-hat nonlocal kernel with $b=1 / 2, \mu^{\prime}=6, s=-1$ and different values for the interaction range $\sigma$ within parameter region 4.

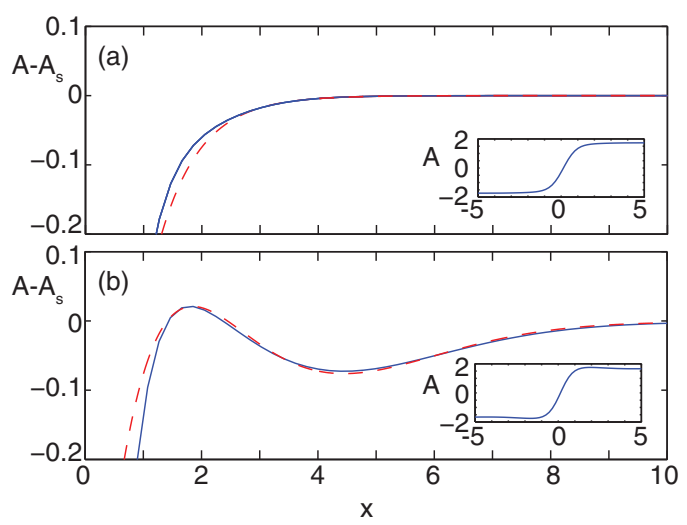

FIG. 13. (Color online) Spatial front profile for the GLE with a Mexican-hat nonlocal kernel for $b=1 / 2, \mu^{\prime}=-6, s=-1$ and (a) $\sigma=1$ and (b) $\sigma=3$. The red dashed lines show the approximations given by Eqs. (32) and (67) (see text).

action with a small interaction range (e.g., $\sigma=1$ ) a similar linear growth is encountered, albeit with a smaller slope, for $d>3$. For smaller values of $d$ the two fronts have a stronger interaction and the logarithm of the velocity increases linearly when decreasing $d$ but at a much slower rate. For $\sigma=1$ although the real part of the complex quartet is not far away from the real doublet, as shown in Fig. 11(d), the separation is still sufficient to warrant a quasimonotonic front shape as shown in Fig. 13(a). Close to the HSS the front profile is well described by an exponential of the form (32) with $q_{1}=-1.433$, which is the value for leading spatial eigenvalue, and fitting the amplitude to $c_{1}=-1.299$. As a consequence, the velocity at which two connected fronts approach each other is a monotonic function of the front separation.

As the range of the nonlocal interaction $\sigma$ increases, the real part of the complex quartet keeps approaching the real doublet as shown in Fig. 11(e) for $\sigma=2$, and a plateau appears in the velocity when the distance is around 3 (see line for $\sigma=2$ in Fig. 12). For $\sigma=3$ clearly the velocity goes to zero at $d=d_{0} \approx 2.3$. Fronts initially separated by a distance larger than this one will approach each other until reaching $d_{0}$ while fronts whose initial distance is slightly smaller than $d_{0}$ will separate until reaching $d_{0}$. Thus, $d_{0}$ is a stable distance at which the fronts lock, forming a LS.

Proceeding in this way one can determine numerically the boundary within region 4 , where the oscillations induced by the complex quartet close to the front core enable the formation of LSs. This boundary is labeled as LO in Fig. 10. For small nonlocal interaction ranges the line $\mathrm{LO}$ approaches to the crossover XR. This comes from the fact that for small values of $\sigma$ the real parts of the doublet and the quartet separate faster than for large values of $\sigma$, as illustrated in Fig. 11 [compare panels (d) and (g), for instance]. Thus, the parameter region where one must account for both the real doublet and the complex quartet is smaller for small $\sigma$.

Figure 13(b) shows an oscillatory front profile for $\sigma=3$ within the parameter region between XR and LO. At difference from the $\mathrm{BD}$ transition here the oscillations appear at a finite spatial wave number and close to the HSS the front profile is very well described by a combination of two exponentials of 
the form

$$
A(x)-A_{s} \approx c_{1} e^{q_{1} x}+c_{2} e^{q_{2} x} \cos \left(k_{2} x+\phi_{2}\right),
$$

where $q_{1}=-0.518$ is the real spatial eigenvalue, while $q_{2}=$ -0.6495 and $k_{2}=0.734$ are the real and imaginary parts of the complex spatial eigenvalue. The coefficients $c_{1}=-0.7014$, $c_{2}=0.9892$, and $\phi_{2}=-1.584$ have been fitted.

\section{CONCLUSIONS}

In this paper we have applied the general framework developed in Part I (see Ref. [2]) to illustrate the effect of nonlocal interactions using the GLE as a prototypical example. In particular, the work presented here allows for a detailed explanation of some of the findings advanced in [1]. One of the main results of [1] was that in spatially extended nonlinear systems exhibiting fronts connecting two equivalent HSSs, the addition of a spatially nonlocal linear interaction term can induce the creation of localized structures in systems with monotonic fronts. This interesting effect is induced by a repulsive nonlocal interaction that is able to induce oscillatory tails in the spatial front profile and, thus, lead to stable LSs. This was shown in Ref. [1] for the case of a Gaussian nonlocal influence kernel. Leveraging the general framework developed in Part I [2], we rationalize these results and show its generality by considering two other choices of the nonlocal influence kernel, a mod-exponential and a Mexican hat. Remarkably, in the case of the two first kernels we have been able to find analytical conditions for the existence of the LSs. In the case of the Mexican-hat kernel, with coexisting attractive and repulsive interactions, LSs are obtained through two different mechanisms for both the cases with short-range excitation and long-range inhibition and the other way around. One mechanism is the $\mathrm{BD}$ transition $[28,35]$ in which oscillations appear initially at infinite wavelength and the other a crossover in the location of the spatial eigenvalues on the complex plain in which finite wavelength oscillations develop.

There are a number of problems that exhibit bistable dynamics and domain walls connecting them and also, presumably, spatially nonlocal effects, e.g., in chemical reactions [36] and in nonlinear optics [37]. The present work shows that spatial nonlocal effects can have a big influence on these phenomena. Comparisons with experimental results can be made more quantitative by reconstructing the experimental kernel $[16,19]$.

\section{ACKNOWLEDGMENTS}

This work was supported by the Belgian Science Policy Office (BeISPO) under Grant No. IAP 7-35, by the Spanish MINECO and FEDER under Projects FISICOS (FIS200760327), DeCoDicA (TEC2009-14101), INTENSE@COSYP (FIS2012-30634), and TRIPHOP (TEC2012-36335), and by Comunitat Autònoma de les Illes Balears. L.G. acknowledges support by the Research Foundation-Flanders (FWO). We thank Professor E. Knobloch and Dr. G. Van der Sande for interesting discussions.

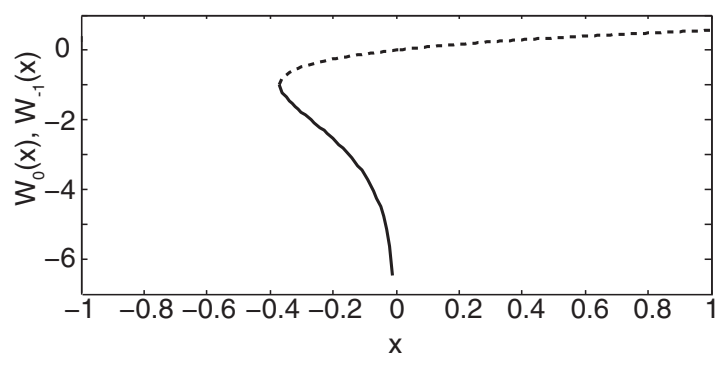

FIG. 14. A plot of the real branches of Lambert's $W$ function, two-valued in the range $[-1 / e, 0]$. The dashed line corresponds to $W_{0}(x)$ and the solid line to $W_{-1}(x)$.

\section{APPENDIX: LAMBERT'S $W$ FUNCTION}

The so-called Lambert's $W$ function [38] is the inverse function of $x=f(y)=y \exp (y)$, i.e., $y=W(x)$ (see, e.g., $[39,40]$ for further details). It can be seen as a generalized logarithm, a useful analogy, because as the (complex) logarithm function, Lambert's $W$ function is multivalued. So, we define it as

$$
x=W_{l}(x) \exp \left[W_{l}(x)\right], \quad l \in \mathbb{Z},
$$

where, in principle, $x \in \mathbb{C}$ and $l \in \mathbb{Z}$ is the branch index. The principal branch, $W_{0}(x)$ or simply $W(x)$, has a branch point at $x=-1 / e$ and a branch cut along the negative real axis $x \in[-\infty,-1 / e]\left[W_{0}(-1 / e)=-1\right]$ and is real valued in the interval $x \in[-1 / e, \infty]$. Moreover, it is analytic at $x=0$, $W_{0}(0)=0$. On the other hand, $W_{-1}(x)$ is real in the interval $x \in[-1 / e, 0]$ and has a branching point at $x=0$ (in addition to the branching at $x=-1 / e$ that it shares with the principal branch). The two real branches of $W$ are shown in Fig. 14.

The Lambert function can also be used to find the exact solution of transcendental equations of the type $x+\exp (x)$ equal to a constant. Thus, a solution to the equation

$$
c x+\exp (a x)=b
$$

can be found with the change

$$
\frac{y}{a}=b-c x
$$

as

$$
\frac{y}{c}=W\left[\frac{a}{c} \exp (a b / c)\right],
$$

and undoing the change of variables one gets

$$
x=\frac{b}{c}-\frac{1}{a} W\left[\frac{a}{c} \exp (a b / c)\right] .
$$

The real branches of Lambert's $W$ function admit the following series expansions, valid close to $x=0[x<0$ for $\left.W_{-1}(x)\right]$,

$$
\begin{gathered}
W_{0}(x)=\sum_{n=1}^{\infty} \frac{(-n)^{n-1}}{n !} x^{n}=x-x^{2}+\frac{3}{2} x^{3}+\cdots, \\
W_{-1}(x)=\ln (-x)-\ln [-\ln (-x)]+\cdots .
\end{gathered}
$$

Equation (A6) corresponds to the Taylor expansion of $W_{0}(x)$ around $x=0$ whose convergence radius is $1 / e$, while (A7) is in fact an asymptotic expansion [39]. 
[1] L. Gelens, D. Gomila, G. Van der Sande, M. A. Matías, and P. Colet, Phys. Rev. Lett. 104, 154101 (2010).

[2] P. Colet, M. A. Matías, L. Gelens, and D. Gomila, Phys. Rev. E 89, 012914 (2014).

[3] F. W. Dabby and J. B. Whinnery, Appl. Phys. Lett. 13, 284 (1968); S. Skupin, M. Saffman, and W. Krolikowski, Phys. Rev. Lett. 98, 263902 (2007); D. Suter and T. Blasberg, Phys. Rev. A 48, 4583 (1993).

[4] T. Lahaye, C. Menotti, L. Santos, M. Lewenstein, and T. Pfau, Rep. Prog. Phys. 72, 126401 (2009).

[5] N. I. Nikolov, D. Neshev, O. Bang, and W. Z. Królikowski, Phys. Rev. E 68, 036614 (2003).

[6] B. K. Esbensen, A. Wlotzka, M. Bache, O. Bang, and W. Krolikowski, Phys. Rev. A 84, 053854 (2011).

[7] C. Conti, M. Peccianti, and G. Assanto, Phys. Rev. Lett. 91, 073901 (2003).

[8] M. G. Clerc, D. Escaff, and V. M. Kenkre, Phys. Rev. E 72, 056217 (2005).

[9] E. Hernández-García, C. López, S. Pigolotti, and K. H. Andersen, Philos. Trans. R. Soc. A 367, 3183 (2009); S. Pigolotti, C. López, E. Hernández-García, and K. H. Andersen, Theor. Ecol. 3, 89 (2010).

[10] M. G. Clerc, D. Escaff, and V. M. Kenkre, Phys. Rev. E 82, 036210 (2010).

[11] W. Krolikowski, O. Bang, J. J. Rasmussen, and J. Wyller, Phys. Rev. E 64, 016612 (2001).

[12] J. Wyller, W. Krolikowski, O. Bang, and J. J. Rasmussen, Phys. Rev. E 66, 066615 (2002).

[13] S. Ouyang, Q. Guo, and W. Hu, Phys. Rev. E 74, 036622 (2006).

[14] G. Bordyugov and H. Engel, Phys. Rev. E 74, 016205 (2006).

[15] B. Ermentrout, Rep. Prog. Phys. 61, 353 (1998).

[16] B. Hellwig, Biol. Cybern. 82, 111 (2000).

[17] S. Coombes, Biol. Cybern. 93, 91 (2005).

[18] S.-I. Shima and Y. Kuramoto, Phys. Rev. E 69, 036213 (2004).

[19] A. Minovich, D. N. Neshev, A. Dreischuh, W. Krolikowski, and Y. S. Kivshar, Opt. Lett. 32, 1599 (2007).

[20] H. R. Wilson and J. D. Cowan, Biophys. J. 12, 1 (1972).

[21] S. Amari, Biol. Cybern. 27, 77 (1977).

[22] M. Hildebrand, H. Skødt, and K. Showalter, Phys. Rev. Lett. 87, 088303 (2001).

[23] D. Mihalache, D. Mazilu, F. Lederer, L.-C. Crasovan, Y. V. Kartashov, L. Torner, and B. A. Malomed, Phys. Rev. E 74, 066614 (2006).

[24] M. Warenghem, J. F. Blach, and J. F. Henninot, J. Opt. Soc. Am. B 25, 1882 (2008).

[25] M. Bache, O. Bang, W. Krolikowski, J. Moses, and F. W. Wise, Opt. Lett. 32, 2490 (2007); M. Bache, O. Bang, J. Moses, and
F. W. Wise, Opt. Express 16, 3273 (2008); B. K. Esbensen, M. Bache, W. Krolikowski, and O. Bang, Phys. Rev. A 86, 023849 (2012).

[26] C. Fernandez-Oto, M. G. Clerc, D. Escaff, and M. Tlidi, Phys. Rev. Lett. 110, 174101 (2013).

[27] M. C. Cross and P. C. Hohenberg, Rev. Mod. Phys. 65, 851 (1993).

[28] R. Devaney, Trans. Amer. Math. Soc. 218, 89 (1976).

[29] J. D. Murray, Mathematical Biology, 3rd ed. (Springer, New York, 2002), Vols. 1 and 2.

[30] J. Swift and P. C. Hohenberg, Phys. Rev. A 15, 319 (1977); B. A. Malomed, A. A. Nepomnyashchy, and M. I. Tribelsky, ibid. 42, 7244 (1990); M. Bestehorn and H. Haken, ibid. 42, 7195 (1990).

[31] J. Burke and E. Knobloch, Chaos 17, 037102 (2007).

[32] L. Gelens, G. Van der Sande, P. Tassin, M. Tlidi, P. Kockaert, D. Gomila, I. Veretennicoff, and J. Danckaert, Phys. Rev. A 75, 063812 (2007); L. Gelens, D. Gomila, G. Van der Sande, J. Danckaert, P. Colet, and M. A. Matías, ibid. 77, 033841 (2008).

[33] This can be shown taking into account that for the argument of $W$ in (31), $\lim _{\sigma \rightarrow \infty}-\exp \left(\frac{\sigma^{2}}{2} \mu^{\prime}-1\right) \rightarrow 0^{-}$. As shown in the Appendix $W_{0}\left(0^{-}\right) \rightarrow 0$, and so $s_{\mathrm{BD}} \rightarrow 0^{-}$, while as $W_{-1}\left(0^{-}\right) \rightarrow$ $-\infty$ it is necessary to use its series expansion. Keeping the first term, $W_{-1}(z) \sim \ln (-z)$ one gets $s_{\mathrm{MI}} \rightarrow\left(2 / \sigma^{2}\right)\left(\sigma^{2} \mu^{\prime} / 2-1\right) \sim$ $\mu^{\prime}$, as shown in Fig. 3.

[34] G. Ermentrout and J. McLeod, Proc. R. Soc. Edinburgh 123A, 461 (1993).

[35] A. J. Homburg and B. Sandstede, in Handbook of Dynamical Systems, edited by B. Hasselblatt, H. Broer, and F. Takens (North Holland, Amsterdam, The Netherlands, 2010), Chap. 8, pp. 379-524.

[36] J. Boissonade, P. D. Kepper, F. Gauffre, and I. Szalai, Chaos 16, 037110 (2006)

[37] A. Esteban-Martín, V. B. Taranenko, J. García, E. Roldán, and G. J. De Valcárcel, Appl. Phys. B 85, 117 (2006); V. B. Taranenko, K. Staliunas, and C. O. Weiss, Phys. Rev. Lett. 81, 2236 (1998).

[38] This special function is quite accessible nowadays as it can be found in modern scientific programs like MATHEMATICA (where it is called ProductLog), MATLAB (where it is called lambertw, and MAPLE (where it is called LambertW).

[39] R. M. Corless, G. H. Gonnet, D. E. G. Hare, D. J. Jeffrey, and D. E. Knuth, Adv. Comput. Math. 5, 329 (1996).

[40] Maplesoft, The Lambert W Function Online help (Maple, Waterloo, Canada, 2012). 